\title{
The affine constrained GNSS attitude model and its multivariate integer least-squares solution
}

\author{
P. J. G. Teunissen
}

Received: 2 September 2011 / Accepted: 12 December 2011 / Published online: 28 December 2011

(C) The Author(s) 2011. This article is published with open access at Springerlink.com

\begin{abstract}
A new global navigation satellite system (GNSS) carrier-phase attitude model and its solution are introduced in this contribution. This affine-constrained GNSS attitude model has the advantage that it avoids the computational complexity of the orthonormality-constrained GNSS attitude model, while it still has a significantly improved ambiguity resolution performance over its unconstrained counterpart. The functional and stochastic model is formulated in multivariate form, for one-, two- and three-dimensional antenna arrays, tracking GNSS signals on an arbitrary number of frequencies with two or more antennas. The stochastic model includes the capability to capture variations in the antennaquality within the array. The multivariate integer leastsquares solution of the model parameters is given and the model's ambiguity success-rate is analysed by means of the ambiguity dilution of precision (ADOP). A general closedform expression for the affine-constrained ADOP is derived, thus providing an easy-to-use and insightful rule-of-thumb for the ambiguity resolution capabilities of the affine constrained GNSS attitude model.
\end{abstract}

Keywords Global navigation satellite systems .

Attitude determination - Affine constrained attitude model . Multivariate integer least squares estimation - Ambiguity dilution of precision

\section{P. J. G. Teunissen}

Department of Spatial Sciences, GNSS Research Centre,

Curtin University of Technology, Bentley, Australia

P. J. G. Teunissen $(\varangle)$

Delft Institute for Earth Observation and Space Systems (DEOS),

Delft University of Technology, Delft, The Netherlands

e-mail: P.J.G.Teunissen@tudelft.nl

\section{Introduction}

GNSS attitude determination is a problem that takes an important place in the development of many navigation, guidance and control systems. It employs multiple GNSS antennas firmly mounted on a platform's body to estimate its orientation with respect to a chosen reference frame. GNSS attitude determination is a rich field of studies, with a wide variety of challenging (terrestrial, sea, air and space) applications (Tu et al. 1996; Bar-Itzhack et al. 1998; Peng et al. 1999; Caporali 2001; Yoon and Lundberg 2002; Park and Teunissen 2003; Li et al. 2004; Simsky et al. 2005). Examples of space applications are satellite formation flying and space platform attitude, guidance and control, see e.g. (Cohen et al. 1994; Axelrad and Ward 1994; Lopes 2002; Ziebart and Cross 2003; Madsen and Lightsey 2004; Dai et al. 2004; Unwin et al. 2002; Buist et al. 2010) and examples of airor shipborne applications are attitude-heading reference systems for aviation, development of antenna pointing systems, joint precision and approach landing, low-cost UAV attitude determination for remote sensing and precise docking of vessels, see e.g. (Corbett 1993; Lu 1995; Lachapelle et al. 1996; DeLorenzo et al. 2004; Hide and Pinchin 2007; Giorgi et al. 2010).

High-precision GNSS attitude determination requires use of the very precise carrier phase observations. However, to fully exploit the high-precision of the carrier phase data, one needs to resolve their unknown double-differenced cycle ambiguities as integers. Once this integer ambiguity resolution has been done successfully, the carrier phase data will act as very precise pseudo range data, thus making precise attitude determination possible.

The earliest methods of attitude ambiguity resolution are the so-called motion-based methods, see e.g. (Cohen and Parkinson 1992; Cohen 1996; Crassidis et al. 1999; Chun 
and Park 1995). These methods take advantage of the change in receiver-satellite geometry induced by platform's motion. They are not applicable, however, on an epoch-by-epoch basis, as the presence of motion is needed per se.

More recently, attitude ambiguity resolution has been solved by searching in the ambiguity domain using the LAMBDA method, see e.g. (Wang et al. 2001; Furuno 2003; Lin et al. 2004; Dai et al. 2004; Monikes et al. 2005; Kuylen et al. 2006, 2005; Psiaki 2006; Hauschild and Montenbruck 2007; Hauschild et al. 2008; Pinchin 2008; Wang et al. 2009). Although this method is known to be efficient and known to maximize the ambiguity success rate, the method has been developed for unconstrained and/or linearly constrained GNSS models (Teunissen 1995, 1999; Verhagen and Teunissen 2006). It is therefore not necessarily optimal for the GNSS attitude determination problem, for which often the antenna geometry or baseline lengths are provided as well.

In order to do proper justice to the a priori information, the nonlinear baseline constraints should be fully integrated into the ambiguity objective function, thereby receiving a proper weighting in its minimization and providing guidance for the integer search. The constrained LAMBDA method is the first method for which this has been achieved. The method's constrained integer least-squares theory has been described in (Teunissen 2010) and its high, single- and multi-baseline, single-epoch success-rate performance has been demonstrated for a variety of land, sea and air experiments in (Giorgi et al. 2010, 2011; Teunissen et al. 2011). But although this method has an increased success-rate performance in comparison with existing techniques, the rigorous inclusion of the nonlinear constraints into the ambiguity objective function has made the complexity of its integer ambiguity search increase as well. This is primarily due to the complexity of its search space, which is now not ellipsoidal anymore. To avoid this complexity, we introduce in this contribution a new alternative method, one that is a compromise between the unconstrained LAMBDA method and its more complex multivariate constrained variant.

The method to be introduced follows from a particular relaxation of the nonlinear constraints. In GNSS attitude models one can generally identify two types of nonlinear constraints, the integer constraints of the carrier-phase ambiguities and the orthonormality constraints of the attitude matrix. These two types of constraints play a distinct role in GNSS attitude determination. The presence of the integer ambiguities enables precise attitude determination, whereas the presence of the orthonormality constraints enables to achieve high ambiguity success-rates and therefore reliable attitude determination. By selecting an affine subset of the orthonormality constraints, the otherwise increased search space complexity can be avoided and standard ambiguity resolution can be applied. The relaxation of the constraints goes, of course, at the expense of model strength and will therefore result in success-rates that are lower than those of the multivariate constrained LAMBDA method. However, when the number of baselines is larger than the dimension of their span, the affine-constrained ambiguity success-rates remain generally high, thus making the easier-to-resolve affine-constrained GNSS attitude model a viable alternative to the orthonormality-constrained GNSS attitude model.

This contribution is organized as follows. In Sect. 2, the GNSS attitude model is introduced in multivariate form. The multivariate formulation is particularly suitable for small GNSS arrays such as used in attitude determination. The functional and stochastic model are given and they are formulated for an arbitrary number of frequencies. The structure of the stochastic model is chosen such that it also can capture variations in antenna-quality within the array. By formulating the parametric orthonormality constraints of the attitude model in implicit form, the attitude constraints are shown to be separable into linear and quadratic constraints. By discarding the quadratic constraints, the affineconstrained multivariate attitude model is obtained. This model is solved in a step-wise manner in Sect. 3. To show the impact of the affine-constraints, the float and fixed leastsquares matrix solutions of the array and ambiguities are derived for both the constrained and unconstrained case. These results are then used as basis for constructing the affine-constrained ambiguity objective function and for the comparison with its unconstrained and orthonormality-constrained counterparts.

In Sect. 4, the ambiguity resolution strength of the affineconstrained GNSS attitude model is described and analysed. This is done by means of the ambiguity dilution of precision (ADOP), for which a general closed form formula is derived. With this general form, we are able to present a very simple and insightful rule-of-thumb describing the model's ambiguity resolution capabilities. This rule clearly shows the role of the various contributing factors of the model, such as phase- and code-precision, number of frequencies and their wavelengths, and number of antennas and satellites. From it the expected ambiguity resolution performances of one-dimensional, two-dimensional and three-dimensional antenna arrays are inferred.

In our multivariate formulation, a frequent use is made of the Kronecker product and the vec-operator. For their properties, see e.g., (Harville 1997; Magnus and Neudecker 1995). In the following, $\mathrm{E}($.$) and \mathrm{D}($.$) denote the operators of expec-$ tation and dispersion. The capital letter $Q$ is reserved for variance matrices. Thus $\mathrm{D}(a)=Q_{a a}$ is the variance matrix of the random vector $a$. Instead of writing $Q_{\operatorname{vec}(Y) \operatorname{vec}(Y)}$ for the variance matrix of $\operatorname{vec}(Y)$, we write $Q_{Y Y}$. Thus $\mathrm{D}(\operatorname{vec}(Y))=$ $Q_{Y Y}$. The squared $M$-weighted norm of a vector $x$ is denoted as $\|x\|_{M}^{2}=x^{\mathrm{T}} M^{-1} x$. 


\section{The multivariate GNSS attitude model}

\subsection{Attitude determination}

The attitude of a rigid body (or platform) is described by the orientation of its body frame with respect to a reference frame. Let there be $r+1$ body points of which one, say the first, is chosen as reference point. Furthermore, let the coordinate-difference vectors, with respect to the reference point, of the remaining $r$ body points be denoted as $b_{\alpha}$, when expressed in the body frame, and as $x_{\alpha}$, when expressed in the reference frame, with $\alpha=1, \ldots, r$. Then the $3 \times r$ baseline matrices $X=\left[x_{1}, \ldots, x_{r}\right]$ and $B=\left[b_{1}, \ldots, b_{r}\right]$ are linked as

$X=R B$

in which $R$ is a rotation matrix, $R \in \mathbb{S O}(3)$. A rotation matrix is an orthonormal matrix, $R^{\mathrm{T}} R=I_{3}$, that has determinant $\operatorname{det}(R)=+1$ (Kuipers 2002). Only three of the rotation matrix entries are independent, since its nine entries fulfill the six conditions of $R^{\mathrm{T}} R=I_{3}$.

The goal of attitude determination is to determine $R$, or parts thereof, from a matrix equation like (1). Once this is done, the attitude parameters can be extracted from its matrix entries. As illustration, consider the Euler-angle parametrization which is commonly used in aerospace applications (Kuipers 2002). Let the reference-frame be the NED (North, East, Down) local level frame and let the (aircraft)bodyframe be defined as having the first axis longitudinal in the direction of flight, the second axis in the direction of the right wing and the third axis completing it as righthanded frame. Then

$R=\left[\begin{array}{ccc}c_{\mathcal{H}} c_{\mathcal{E}} & -s_{\mathcal{H}} c_{\mathcal{B}}+c_{\mathcal{H}} s_{\mathcal{E}} s_{\mathcal{B}} & s_{\mathcal{H}} s_{\mathcal{B}}+c_{\mathcal{H}} s_{\mathcal{E}} c_{\mathcal{B}} \\ s_{\mathcal{H}} c_{\mathcal{E}} & c_{\mathcal{H}} c_{\mathcal{B}}+s_{\mathcal{H}} s_{\mathcal{E}} s_{\mathcal{B}} & -c_{\mathcal{H}} s_{\mathcal{B}}+s_{\mathcal{H}} s_{\mathcal{E}} c_{\mathcal{B}} \\ -s_{\mathcal{E}} & c_{\mathcal{E}} s_{\mathcal{B}} & c_{\mathcal{E}} c_{\mathcal{B}}\end{array}\right]$

with Euler-angles: heading $\mathcal{H}$, elevation $\mathcal{E}$ and bank $\mathcal{B}$ (we used the short-hand notation $c_{\alpha}=\cos (\alpha)$ and $s_{\alpha}=\sin (\alpha)$ ). Hence, once $R$ is known, the Euler angles can be determined from the rotation matrix entries $R_{21}, R_{31}$ and $R_{32}$ as

$$
\begin{aligned}
\mathcal{H} & =\arcsin \left(\frac{R_{21}}{\cos (\mathcal{E})}\right), \mathcal{E}=-\arcsin \left(R_{31}\right) \\
\mathcal{B} & =\arcsin \left(\frac{R_{32}}{\cos (\mathcal{E})}\right)
\end{aligned}
$$

Thus heading $\mathcal{H}$ and elevation $\mathcal{E}$ can already be determined from the first column vector of $R$, while for the bank angle $\mathcal{B}$ the complete rotation matrix must be determinable.

It is not always possible to determine the complete $3 \times 3$ attitude matrix $R$. It depends on the span of the baselines and thus on the rank of $X$, whether or not $R$ can be fully or partially recovered. For the rank of $X$ we have
$1 \leq \operatorname{rank}(X)=\operatorname{rank}(B)=q \leq \min (3, r)$

The two baseline matrices $X$ and $B$ have the same rank, since $R$ has full column rank. The baselines achieve their full span if $q=\min (3, r)$. In that case, one baseline spans $\mathbb{R}^{1}$, two baselines span $\mathbb{R}^{2}$ and three or more baselines span $\mathbb{R}^{3}$. A less than full span is achieved if $q<\min (3, r)$, in which case matrix $B$ of (1) is rank deficient.

In order to avoid working with a rank deficient matrix $B$, as might happen with formulation (1), we will work from now on with the formulation

$X=R B, X \in \mathbb{R}^{3 \times r}, R \in \mathbb{O}^{3 \times q}, B \in \mathbb{R}^{q \times r}$

in which $\mathbb{0}^{3 \times q}$ denotes the set of $3 \times q$ matrices of which the $q$ column vectors form an orthonormal span. The difference between this formulation and that of (1) is that now the number of orthonormal columns of $R$ has been set equal to the rank of $X$. This formulation has thus eliminated the indeterminable part of $R$ in (1) when rank of $X$ is less than $\min (3, r)$. That the same symbols are used in (1) and (5) should not pose a problem. If $q=3$, then there is no difference between (1) and (5).

In case of GNSS attitude determination, matrix $X$ is estimated from GNSS data, while matrix $B$, describing the relative geometry of the $r+1$ body points in the body frame, is assumed given. Once a GNSS-estimate of $X$ is available, say $\check{X}$, an estimate of the attitude-matrix $R$ can be determined in a least-squares (LS) sense as

$\check{R}=\arg \min _{R \in \mathbb{O}^{3 \times q}}\|\operatorname{vec}(\check{X}-R B)\|_{Q_{\check{X} \check{X}}}^{2}$

This problem can be solved analytically in case $Q_{\check{X} \check{X}}$ is a scaled unit matrix, see e.g. (Wahba 1965; Schonemann 1966; Wertz 1984). In our present case, however, the variance matrix will be fully populated, thus requiring that the nonlinear least-squares problem (6) is solved using one of the iterative descent methods, like the Gauss-Newton method, having a linear rate of convergence, or the Newton method, having a quadratic rate of convergence, see e.g. (Teunissen 1990). The analytical solution of 'Wahba's problem' may then serve as initialization of the iteration.

In order to determine a precise attitude $\check{R}$, the antennageometry $B$ needs to be properly chosen and the input $\check{X}$ (cf. 6) needs to be as precise as possible. To realize the most precise $\check{X}$ with GNSS, requires the use of its very precise carrier phase data. However, to fully exploit the high-precision of the carrier phase data, one needs to be able to successfully resolve their unknown double-differenced cycle ambiguities as integers. Integer ambiguity resolution plays therefore a pivotal role in precise GNSS attitude determination. 


\subsection{The GNSS array model}

We now formulate the GNSS array model and show how the GNSS observables can be linked to matrix $X$ of (5). We assume that the body is equipped with a body-fixed array of $r+1$ GNSS antennas all tracking the same $s+1$ satellites on the same $f$ frequencies. The geometry of the relative antenna positions in the body-frame is assumed given by the $q \times r$ matrix $B$ of (5). Furthermore, we assume the array-size to be such that the differential atmospheric delays (troposphere and ionosphere) between the antennas can be neglected. With these assumptions, we can formulate the single-epoch, multifrequency GNSS array model in multivariate form as

$\mathrm{E}(Y)=M X+N Z, \quad X \in \mathbb{R}^{3 \times r}, Z \in \mathbb{Z}^{f s \times r}$

with $Y$ the $2 f s \times r$ double-differenced (DD) data matrix, $(M, N)$ the $2 f s \times(3+f s)$ design matrix, $X \in \mathbb{R}^{3 \times r}$ the unknown real-valued baseline matrix and $Z \in \mathbb{Z}^{f s \times r}$ the unknown integer ambiguity matrix. The carrier phase and pseudo range data matrix is structured as $Y=\left[Y_{\phi}^{\mathrm{T}}, Y_{p}^{\mathrm{T}}\right]^{\mathrm{T}}$, with $Y_{\phi}=\left[y_{\phi ; 1}, \ldots, y_{\phi ; r}\right]$ and $Y_{p}=\left[y_{p ; 1}, \ldots, y_{p ; r}\right]$, where $y_{\phi ; \alpha}=\left[y_{\phi ; \alpha, 1}^{\mathrm{T}}, \ldots, y_{\phi ; \alpha, f}^{\mathrm{T}}\right]^{\mathrm{T}}$ and $y_{p ; \alpha}=\left[y_{p ; \alpha, 1}^{\mathrm{T}}, \ldots\right.$, $\left.y_{p ; \alpha, f}^{\mathrm{T}}\right]^{\mathrm{T}}$ are the multi-frequency $f s \times 1$ vectors of DD phase and code observables of baseline $\alpha$. For the entries of the design matrix $(M, N)$, we have $M=\left(e_{2 f} \otimes G\right)$ and $N=\left(L \otimes I_{S}\right)$, with $G$ the $s \times 3$ matrix of unit direction vectors that capture the DD relative receiver-satellite geometry and $2 f \times f$ matrix $L=\left[\Lambda^{\mathrm{T}}, 0^{\mathrm{T}}\right]^{\mathrm{T}}$, with $\Lambda=\operatorname{diag}\left(\lambda_{1}, \ldots, \lambda_{f}\right)$ the diagonal wavelength matrix, having the entries $\lambda_{j}=$ $c / f_{j}, j=1, \ldots, f$ ( $c$ is the speed of light; $f_{j}$ is the $j$ th frequency).

Note that the above multivariate formulation assumes the same geometry matrix $G$ for all baselines in the array. Hence, it is assumed that all array antennas 'see' the same satellite in the same direction. This approximation is good enough for our application, since the array size is assumed sufficiently small (e.g., less than $100 \mathrm{~m}$ ) in relation to the high altitudes of GNSS satellites (about 20,000 km).

The dispersion of the GNSS array data matrix is assumed given by the $2 f s r \times 2 f s r$ variance matrix

$\mathrm{D}(\operatorname{vec}(Y))=P \otimes Q$

where

$P=D_{r}^{\mathrm{T}} Q_{r} D_{r}, Q=\operatorname{blockdiag}\left(Q_{\Phi}, Q_{P}\right)$

$Q_{\Phi}=Q_{f} \otimes D_{s}^{\mathrm{T}} Q_{\phi} D_{s}, Q_{P}=Q_{f} \otimes D_{s}^{\mathrm{T}} Q_{p} D_{s}$

The matrices $Q_{r}, Q_{f}, Q_{\phi}$ and $Q_{p}$ are co-factor matrices and the matrices $D_{r}^{\mathrm{T}}$ and $D_{s}^{\mathrm{T}}$ are differencing matrices. With the $(s+1) \times(s+1)$ matrices $Q_{\phi}$ and $Q_{p}$, the relative precision contribution of the undifferenced phase and code data is captured. They may also include the satellite elevation dependency of dispersion, by for instance, in case of $Q_{\phi}$, using the diagonal matrix $Q_{\phi}=\sigma_{\phi}^{2} \operatorname{diag}\left[\sin ^{-2}\left(\epsilon_{1}\right), \ldots, \sin ^{-2}\left(\epsilon_{s+1}\right)\right]$, with $\epsilon_{i}$ being the elevation of satellite $i$ and $\sigma_{\phi}^{2}$ being the undifferenced phase variance at zenith.

The $s \times(s+1)$ differencing matrix $D_{s}^{\mathrm{T}}$ transforms undifferenced observables into between-satellite single-differenced observables. For example, if the last satellite is taken as reference (pivot), then $D_{s}^{\mathrm{T}}=\left[I_{s},-e_{s}\right]$. The matrices $Q_{f}$ and $Q_{r}$ capture the dispersion contribution of the different frequencies and different antenna/receivers. For instance, if the array is equipped with antennas of different quality, then the $(r+1) \times(r+1)$ matrix $Q_{r}$ can be used to capture the relative receiver quality. The $r \times(r+1)$ differencing matrix $D_{r}^{\mathrm{T}}$ transforms between-satellite single-differenced observables into DD observables. For example, if the first antenna is taken as reference (master), then $D_{r}^{\mathrm{T}}=\left[-e_{r}, I_{r}\right]$.

2.3 The nonlinearity of the GNSS compass and attitude model

The multivariate GNSS attitude model is formed by the equations (5), (7) and (8). It is a nonlinear model due to the presence of the orthonormal matrix $R$ in (5). This nonlinearity is of a quadratic nature as can be seen when eliminating $R$ from (5) using $R^{\mathrm{T}} R=I_{q}$.

\subsubsection{Quadratic compass model}

To get some appreciation for the nonlinearity involved, we first consider the single-baseline case. For $r=1$, we have $x^{\mathrm{T}} x=b^{\mathrm{T}} b=c(X=x, B=b)$. Hence, the multivariate model reduces then to the univariate, quadratically constrained, single-baseline model

$\mathrm{E}(y)=M x+N z, x^{\mathrm{T}} x=c, \mathrm{D}(y)=P Q$

with $y \in \mathbb{R}^{2 f s}, x \in \mathbb{R}^{3}$ and $z \in \mathbb{Z}^{f s}$. $P$ is now a scalar. With $Q_{r}=I_{2}$ (two receivers of same quality) its value is $P=$ $[-1,1][-1,1]^{\mathrm{T}}=2$. Model $(10)$ is known as the nonlinear GNSS Compass model (Park and Teunissen 2009; Teunissen 2010). Once $x$ has been ambiguity resolved, precise estimates of heading $\mathcal{H}$ and elevation $\mathcal{E}$ of the baseline can be determined. The quadratic constraint in (10) is a length constraint on the baseline vector. It implies that the baseline vector is constrained to lie on a sphere with known radius, $\mathbb{S}=\left\{x \in \mathbb{R}^{3} \mid\|x\|=\sqrt{c}\right\}$.

To determine the solution of (10) in a least-squares (LS) sense, we need to solve the quadratically-constrained (mixed) integer least-squares (QC-ILS) problem

$$
\begin{aligned}
& \min _{x \in \mathbb{S}, z \in \mathbb{Z}^{f s}}\|y-M x-N z\|_{P Q}^{2}=\|\hat{e}\|_{P Q}^{2} \\
& +\min _{z \in \mathbb{Z}^{f s}}\left(\|\hat{z}-z\|_{Q_{\hat{z} \hat{z}}}^{2}+\min _{x^{\mathrm{T}} x=c}\|\hat{x}(z)-x\|_{Q_{\hat{x}(z) \hat{x}(z)}^{2}}\right)
\end{aligned}
$$


in which $\hat{e}=y-M \hat{x}-N \hat{z}$ is the LS-residual vector of the unconstrained model, i.e. model (10) without integer and quadratic constraints; $\hat{x}$ and $\hat{z}$ are the unconstrained minimizers of $\|y-M x-N z\|_{P Q}^{2}$, having variance-covariance matrices $Q_{\hat{x} \hat{x}}, Q_{\hat{x} \hat{z}}$ and $Q_{\hat{z} \hat{z}}$, and $\hat{x}(z)=\hat{x}-Q_{\hat{x} \hat{z}} Q_{\hat{z} \hat{z}}^{-1}(\hat{z}-z)$ is the conditional LS-solution having $Q_{\hat{x}(z) \hat{x}(z)}=Q_{\hat{x} \hat{x}}-$ $Q_{\hat{x} \hat{z}} Q_{\hat{z} \hat{z}}^{-1} Q_{\hat{z} \hat{x}}$ as variance matrix. The dependence on $z$ is made explicit through the argument in the notation of $\hat{x}(z)$.

If we denote the minimizer of the third term on the right hand side of (11) as $\check{x}(z)=\arg \min _{x \in \mathbb{S}}\|\hat{x}(z)-x\|_{Q_{\hat{x}(z) \hat{x}(z)}}^{2}$, then the minimizers $\check{z} \in \mathbb{Z}^{f s}$ and $\check{x} \in \mathbb{R}^{3}$ of the QC-ILS problem (11) follow as

$\check{z}=\arg \min _{z \in \mathbb{Z}^{f s}} F(z)$ and $\check{x}=\check{x}(\check{z})$

with the ambiguity objective function given as

$F(z)=\|\hat{z}-z\|_{Q_{\hat{z} \hat{z}}}^{2}+\|\hat{x}(z)-\check{x}(z)\|_{Q_{\hat{x}(z) \hat{x}(z)}}^{2}$

This is a nonstandard ambiguity objective function. It is nonstandard due to the presence of the second term on the right hand side of (13). This term is absent in case one would estimate the integer ambiguities of model (10) without taking the quadratic constraint into account. In that case the ambiguity objective function would be given as $E(z)=\|\hat{z}-z\|_{Q_{\hat{z} \hat{z}}}^{2}$.

To appreciate the additional complexity that the quadratic constraint of the GNSS compass model brings to integer ambiguity resolution, we compare the two ambiguity objective functions $F(z)$ and $E(z)$. First note that $F(z)$, in contrast to $E(z)$, not only depends on the ambiguity solution $\hat{z}$, but also on the baseline solution $\hat{x}$. Thus both $\hat{z}$ and $\hat{x}$ are needed as input for the integer minimizer of $F(z)$.

The term by which $F(z)$ differs from $E(z)$, measures the distance, in the metric of $Q_{\hat{x}(z) \hat{x}(z)}$, between $\hat{x}(z)$ and the sphere $\mathbb{S}$. Hence, potential integer candidates $z \in \mathbb{Z}^{f s}$ are now not only downweighted if they are further away from the float solution $\hat{z}$, as is the case with $E(z)$, but also if their corresponding conditional baseline $\hat{x}(z)$ is further apart from the sphere $\mathbb{S}$. As demonstrated in the experiments of (Teunissen et al. 2011), it is this additional penalty in the objective function that allows for the much higher success rates as compared to the success rates obtained with the unconstrained model.

This much improved performance comes at the cost of having to solve a more complex problem. The term by which $F(z)$ differs from $E(z)$, makes $F(z)$, in contrast to $E(z)$, a nonquadratic function in $z$. Hence, its search space, $\Omega_{F}=$ $\left\{z \in \mathbb{Z}^{f s} \mid F(z) \leq \chi^{2} \in \mathbb{R}^{+}\right\}$, will not be ellipsoidal, thus complicating the integer search for $\check{z}$. To cope with this additional complexity, two different search strategies have been developed, an expansion and a shrinkage strategy, see (Park and Teunissen 2009; Teunissen 2010).

Not only are the contour surfaces of $F(z)$ more complex than those of $E(z)$, also the evaluation of $F(z)$ is more time consuming than that of $E(z)$. The presence of the second term in (13) implies that $\check{x}(z)$ needs to be computed every time $F(z)$ is evaluated. Since the computation of the nonlinear least-squares solution $\check{x}(z)$ is already nontrivial by itself (geometrically it can be depicted as the point where the ellipsoid $\mathbb{E}=\left\{x \in \mathbb{R}^{3} \mid \quad\|\hat{x}(z)-x\|_{Q_{\hat{x}(z) \hat{x}(z)}}^{2}=\right.$ constant $\}$ just touches the sphere $\mathbb{S}$ ), the presence of this second term is a potential threat for the computational efficiency of the integer search. In the constrained LAMBDA method this problem is solved by making use of easier-to-compute bounding functions of $F(z)$.

\subsubsection{Quadratic attitude model}

The multivariate generalization of (10) follows by setting $X^{\mathrm{T}} X=B^{\mathrm{T}} B=C$. This gives the multivariate, quadratically constrained (mixed) integer linear model

$\mathrm{E}(Y)=M X+N Z, X^{\mathrm{T}} X=C, \mathrm{D}(\operatorname{vec}(Y))=P \otimes Q$

with $Y \in \mathbb{R}^{2 f s \times r}, X \in \mathbb{R}^{3 \times r}$ and $Z \in \mathbb{Z}^{f s \times r}$. This model formulation is equivalent to that of the general multivariate GNSS attitude model (cf. 5, 7 and 8) for $q=r \leq 3$, that is, when $B$ is invertible.

In analogy with (12) and (13), one can show (cf. 38) that the QC-ILS solution of the multivariate model (14) is given as

$\check{Z}=\arg \min _{Z \in \mathbb{Z}^{f s \times r}} H(Z)$ and $\check{X}=\check{X}(\check{Z})$

in which the multivariate ambiguity objective function is given as

$H(Z)=\|\operatorname{vec}(\hat{Z}-Z)\|_{Q_{\hat{Z} \hat{Z}}}^{2}+\|\operatorname{vec}(\hat{X}(Z)-\check{X}(Z))\|_{Q_{\hat{X}(Z) \hat{X}(Z)}}^{2}$

where

$\check{X}(Z)=\arg \min _{X^{\mathrm{T}} X=C}\|\operatorname{vec}(\hat{X}(Z)-X)\|_{Q_{\hat{X}(Z) \hat{X}(Z)}^{2}}^{2}$

Again the presence of the second, nonquadratic, term in the ambiguity objective function (cf. 16) results in a much improved success-rate performance as compared to both the unconstrained model and the single-baseline constrained model (10). The excellent success-rate performance of the multivariate ambiguity objective function (16) has been demonstrated for single-frequency, epoch-by-epoch, GNSS-only attitude determination in (Giorgi et al. 2010, 2011). The presence of the non-quadratic term in (16) also increases the computational complexity, which in the multivariate constrained LAMBDA method is taken care of through the use of multivariate bounding functions (Giorgi et al. 2011).

\subsection{Linear and quadratic constraints}

The quadratically constrained model (14) is only equivalent to the general nonlinear GNSS attitude model (cf. 5, 7, 8) 
if matrix $B$ is square and invertible, that is, if $q=r \leq 3$. Although such dual-, triple- and quadrupel-antenna configurations do cover a large part of all GNSS attitude applications, they do not cover all of them. Antenna geometries that are not covered by $q=r \leq 3$, are, for instance, 'degenerate' configurations where all of the three or more antennas are placed in a straight line $(q=1, r>1)$, or where all of the four or more antennas are placed in a plane $(q=2, r>2)$. But also all multi-antenna configurations that consist of more than four antennas $(r>3)$ are not covered by formulation (14).

The following lemma gives a complete description of the constraints involved in the general GNSS attitude model (cf. $5,7,8)$, thus also of the extra constraints that occur when either $q<\min (3, r)$ or $r>3$ is true.

Lemma 1 (Orthonormal matrix parametrization) Let $X$ be a $3 \times r$ matrix and $B$ be $a q \times r$ matrix of $\operatorname{rank}(B)=q$. Then the equivalent implicit form of the matrix equation

$X=R B, \quad R \in \mathbb{O}^{3 \times q}$

is given by

$X S=0$ and $(X T)^{\mathrm{T}}(X T)=I_{q}$

where $S$ is an $r \times(r-q)$ basis matrix of the null space of $B$ $(B S=0)$ and $T$ is an $r \times q$ right-inverse of $B\left(B T=I_{q}\right)$.

Proof The proof is given in the Appendix.

This lemma shows that in addition to the quadratic constraints, also linear constraints may be present. The total number of constraints described by the orthonormal matrix parametrization (18) is equal to $\frac{1}{2} q(q-5)+3 r$. Of these constraints, $3(r-q)$ are linear and $\frac{1}{2} q(q+1)$ are quadratic. There are at most six quadratic constraints $(q=3)$. There is only one quadratic constraint if $q=1$ and there are three quadratic constraints if $q=2$. The linear constraints are only present when the number of baselines is larger than the dimension of their span, $r>q$. This means for linear constraints to be present, that more than two antennas are needed in case the antennas are all placed along a straight line and that more than three antennas are needed in case the antennas are all placed in a plane. In all other cases, more than four antennas will be needed. And in such cases, each additional single antenna will introduce three extra linear constraints.

\section{The affine constrained GNSS attitude model}

\subsection{The affine model formulation}

If we neglect the $\frac{1}{2} q(q+1)$ quadratic constraints of (19) and thus only take the $3(r-q)$ linear constraints of $X S=0$ into account, the multivariate GNSS attitude model takes the linear form

$\mathrm{E}(Y)=M X+N Z, X S=0, \mathrm{D}(\operatorname{vec}(Y))=P \otimes Q$ with $Y \in \mathbb{R}^{2 f s \times r}, M \in \mathbb{R}^{2 f s \times 3}, N \in \mathbb{R}^{2 f s \times f s}, S \in$ $\mathbb{R}^{r \times(r-q)}, P \in \mathbb{R}^{r \times r}, Q \in \mathbb{R}^{2 f s \times 2 f s}$ and the unknown parameter matrices $X \in \mathbb{R}^{3 \times r}$ and $Z \in \mathbb{Z}^{f s \times r}$. This model will be referred to as the affine constrained GNSS attitude model, since the linear matrix constraint $X S=0$ implies the formulation of an affine transformation between bodyand reference-frame. This can be seen as follows. Since the orthogonal complement of the null space of a matrix is equal to the range space of its transpose, $\mathcal{N}(X)^{\perp}=\mathcal{R}\left(X^{\mathrm{T}}\right)$, it follows from $S$ being a basis matrix of $\mathcal{N}(X)$ and $B^{\mathrm{T}}$ being a basis matrix of $\mathcal{R}\left(X^{\mathrm{T}}\right)$, that we have the equivalence

$X S=0 \Longleftrightarrow X=R B$ for some $R \in \mathbb{R}^{3 \times q}$

Hence, working with the implicit matrix constraint $X S=0$ is equivalent to dropping the orthonormality constraints on $R$, thus effectively turning the transformation $X=R B$ into an affine transformation.

In the following, we work interchangeably with the implicit formulation (20) and with its parametric counterpart

$\mathrm{E}(Y)=M X+N Z, X=R B, \mathrm{D}(\operatorname{vec}(Y))=P \otimes Q$

in which the affine-attitude matrix $R \in \mathbb{R}^{3 \times q}$ can replace the baseline matrix $X \in \mathbb{R}^{3 \times r}$ as the unknown parameter matrix. The LS-solutions of (22) can also be used to study the performance of the weaker unconstrained model. By substituting $B=I$ in the LS-solutions of the affine-constrained model, one automatically obtains the corresponding unconstrained LS-solutions.

The LS-solution of model (20) is defined as

$$
\begin{aligned}
{\left[\check{X}_{c}^{\mathrm{T}}, \check{Z}_{c}^{\mathrm{T}}\right]^{\mathrm{T}}=} & \underset{X \in \mathbb{R}^{3 \times r}, Z \in \mathbb{Z}^{f s \times r}, X S=0}{\arg \min } \\
& \times\left\|\operatorname{vec}\left(Y-[M, N]\left[X^{\mathrm{T}}, Z^{\mathrm{T}}\right]^{\mathrm{T}}\right)\right\|_{Q_{Y Y}}^{2}
\end{aligned}
$$

Note that there are two types of constraints involved. The linear constraints on the baseline matrix $X(X S=0)$ and the integer constraints on the ambiguity matrix $Z\left(Z \in \mathbb{Z}^{f s \times r}\right)$. Although both type of constraints play an important role in strengthening the attitude model, the role they have to play is different. As it is shown in the next sections, the primary purpose of the integer constraints is to be able to determine a precise attitude solution, while that of the linear constraints is to ensure that this can be done with sufficient probability of success.

Also note that the affine constraints are taken as 'hard' constraints. It is therefore assumed that the a-priori determination of the body-frame baseline matrix $B$, or at least of its null space, can be done with negligible uncertainty. Would this not be the case, then a weighted approach, somewhat similar to the one given in (Teunissen 2010), will need to be followed.

In order to determine $\check{X}_{c}$ and $\check{Z}_{c}$ of (23), we first determine the so-called 'float' and 'fixed' solutions. The LS-solutions 
of (20) and (22) are referred to as 'float' solutions if they are obtained assuming $Z$ to be real-valued instead of integer-valued. The LS baseline matrix solution is referred to as a 'fixed' solution if it is obtained assuming $Z$ known. First we derive the 'float' solutions.

\subsection{Unconstrained and constrained float baseline matrix}

The affine-constrained float LS-solutions of model (22) are denoted as $\hat{R}_{c}$ and $\hat{Z}_{c}$, respectively. They follow from solving the system of normal equations,

$$
\begin{aligned}
& {\left[\begin{array}{cc}
B P^{-1} B^{\mathrm{T}} \otimes M^{\mathrm{T}} Q^{-1} M & B P^{-1} \otimes M^{\mathrm{T}} Q^{-1} N \\
P^{-1} B^{\mathrm{T}} \otimes N^{\mathrm{T}} Q^{-1} M & P^{-1} \otimes N^{\mathrm{T}} Q^{-1} N
\end{array}\right]\left[\begin{array}{c}
\operatorname{vec}\left(\hat{R}_{c}\right) \\
\operatorname{vec}\left(\hat{Z}_{c}\right)
\end{array}\right]} \\
& =\left[\begin{array}{c}
\left(B P^{-1} \otimes M^{\mathrm{T}} Q^{-1}\right) \operatorname{vec}(Y) \\
\left(P^{-1} \otimes N^{\mathrm{T}} Q^{-1}\right) \operatorname{vec}(Y)
\end{array}\right]
\end{aligned}
$$

First we consider $\hat{R}_{c}$. After reduction of the above normal equations, we obtain the reduced normal equations

$$
\begin{gathered}
{\left[B P^{-1} B^{\mathrm{T}} \otimes \bar{M}^{\mathrm{T}} Q^{-1} \bar{M}\right] \operatorname{vec}\left(\hat{R}_{c}\right)} \\
\quad=\left[B P^{-1} \otimes \bar{M}^{\mathrm{T}} Q^{-1}\right] \operatorname{vec}(Y)
\end{gathered}
$$

with $\bar{M}=P_{N}^{\perp} M$ and the projectors $P_{N}^{\perp}=I_{2 f s}-P_{N}$ and $P_{N}=N\left(N^{\mathrm{T}} Q^{-1} N\right)^{-1} N^{\mathrm{T}} Q^{-1}$. From the reduced normal system (25), the LS-solution $\hat{R}_{c}$ and its variance matrix $Q_{\hat{R}_{c} \hat{R}_{c}}=\mathrm{D}\left(\operatorname{vec}\left(\hat{R}_{c}\right)\right)$ follow as

$$
\begin{aligned}
& \hat{R}_{c}=\left[\left(\bar{M}^{\mathrm{T}} Q^{-1} \bar{M}\right)^{-1} \bar{M}^{\mathrm{T}} Q^{-1}\right][Y]\left[P^{-1} B^{\mathrm{T}}\left(B P^{-1} B^{\mathrm{T}}\right)^{-1}\right] \\
& Q_{\hat{R}_{c} \hat{R}_{c}}=\left(B P^{-1} B^{\mathrm{T}}\right)^{-1} \otimes\left(\bar{M}^{\mathrm{T}} Q^{-1} \bar{M}\right)^{-1}
\end{aligned}
$$

From these expressions, the unconstrained solution is easily obtained by setting $B=I$. In doing so, we find that the unconstrained baseline-matrix solution $\hat{X}$ and constrained affine-attitude solution $\hat{R}_{c}$, together with their variance matrices, are given and linked as

$$
\begin{aligned}
& \hat{X}=\left(\bar{M}^{\mathrm{T}} Q^{-1} \bar{M}\right)^{-1} \bar{M}^{\mathrm{T}} Q^{-1} Y, Q_{\hat{X} \hat{X}}=\frac{1}{2} P \otimes Q_{\hat{x} \hat{x}} \\
& \hat{R}_{c}=\hat{X} P^{-1} B^{\mathrm{T}}\left(B P^{-1} B^{\mathrm{T}}\right)^{-1}, Q_{\hat{R}_{c} \hat{R}_{c}}=\frac{1}{2} K \otimes Q_{\hat{x} \hat{x}}
\end{aligned}
$$

where $K=\left(B P^{-1} B^{\mathrm{T}}\right)^{-1}$ and $Q_{\hat{x} \hat{x}}=2\left(\bar{M}^{\mathrm{T}} Q^{-1} \bar{M}\right)^{-1}$ is the variance matrix of a float single-baseline solution (assuming its two antennas of equal quality). The factor $\frac{1}{2}$ in (27) compensates for the factor 2 of $Q_{\hat{x} \hat{x}}$.

Note that the expressions of (27) facilitate parallel processing. The data of each baseline is mapped by the same matrix $\left(\bar{M}^{\mathrm{T}} Q^{-1} \bar{M}\right)^{-1} \bar{M}^{\mathrm{T}} Q^{-1}$ to the baseline matrix $\hat{X}$, while the coordinates of each of its baseline vectors is mapped by the same matrix $P^{-1} B^{\mathrm{T}}\left(B P^{-1} B^{\mathrm{T}}\right)^{-1}$ to the column vectors of $\hat{R}_{c}$. Also note that the contributions from the receiver-satellite geometry (via $M$ ) and the antenna-array geometry (via $B$ ) are clearly visible in the dispersion of $\hat{R}_{c}$. Although the receiversatellite dispersion contribution will be difficult to manipulate by a user, the contribution of the antenna-geometry can be changed so as to obtain a favourable contribution to the dispersion of $\hat{R}_{c}$.

The effect that the affine constraints have on the baseline matrix, can be made explicit by substituting the expression for $\hat{R}_{c}$ (cf. 27) into $\hat{X}_{c}=\hat{R}_{c} B$. This gives

$$
\hat{X}_{c}=\hat{X} P_{S}^{\perp}, \quad Q_{\hat{X}_{c} \hat{X}_{c}}=\frac{1}{2} P P_{S}^{\perp} \otimes Q_{\hat{x} \hat{x}}
$$

where $P_{S}^{\perp}=P^{-1} B^{\mathrm{T}}\left(B P^{-1} B^{\mathrm{T}}\right)^{-1} B$. The projector $P_{S}^{\perp}$ can be expressed in $S$ as $P_{S}^{\perp}=I_{r}-P_{S}$, where $P_{S}=$ $S\left(S^{\mathrm{T}} P S\right)^{-1} S^{\mathrm{T}} P$. Note that $(28)$ is the solution one gets when solving the multivariate linear model of condition equations $\mathrm{E}(\hat{X} S)=0, \mathrm{D}(\operatorname{vec}(\hat{X}))=\frac{1}{2} P \otimes Q_{\hat{x} \hat{x}}$ in a LS-sense.

\subsection{Unconstrained and constrained float ambiguity matrix}

Now we consider the estimation of the ambiguity matrix $Z$. First we determine the unconstrained float ambiguity estimator $\hat{Z}$. This matrix estimator and its variance matrix follow from solving the normal equations (24) for $B=I$ as

$$
\hat{Z}=\left(\bar{N}^{\mathrm{T}} Q^{-1} \bar{N}\right)^{-1} \bar{N}^{\mathrm{T}} Q^{-1} Y, \quad Q_{\hat{\mathrm{Z}} \hat{z}}=\frac{1}{2} P \otimes Q_{\hat{z} \hat{z}}
$$

in which $Q_{\hat{z} \hat{z}}=2\left(\bar{N}^{\mathrm{T}} Q^{-1} \bar{N}\right)^{-1}$ is the single-baseline ambiguity variance matrix and where $\bar{N}=P_{M}^{\perp} N$, with projectors $P_{M}^{\perp}=I_{2 f s}-P_{M}$ and $P_{M}=M\left(M^{\mathrm{T}} Q^{-1} M\right)^{-1} M^{\mathrm{T}} Q^{-1}$.

To determine the constrained float ambiguity estimator, again the normal equations (24) can be used. However, in order to make the difference between $\hat{Z}_{c}$ and $\hat{Z}$ explicit, it is more instructive to use the relation

$\operatorname{vec}\left(\hat{Z}_{c}\right)=\operatorname{vec}(\hat{Z})-Q_{\hat{Z} \hat{X}} Q_{\hat{X} \hat{X}}^{-1} \operatorname{vec}\left(\hat{X}-\hat{X}_{c}\right)$

Since $Q_{\hat{Z} \hat{X}} Q_{\hat{X} \hat{X}}^{-1}=I_{r} \otimes Q_{\hat{z} \hat{x}} Q_{\hat{x} \hat{x}}^{-1}$ and $\hat{X}-\hat{X}_{c}=\hat{X} P_{S}$ (cf. 28), it follows that

$\hat{Z}_{c}=\hat{Z}-Q_{\hat{z} \hat{x}} Q_{\hat{x} \hat{x}}^{-1} \hat{X} P_{S}$

$Q_{\hat{Z}_{c} \hat{Z}_{c}}=\frac{1}{2} P \otimes Q_{\hat{z} \hat{z}}-\frac{1}{2} P P_{S} \otimes Q_{\hat{z} \hat{x}} Q_{\hat{x} \hat{x}}^{-1} Q_{\hat{x} \hat{z}}$

This result clearly shows the contribution of the affine constraints to the float ambiguity solution. From comparing (29) with (31), it follows that $Q_{\hat{Z} \hat{Z}}-Q_{\hat{Z}_{c} \hat{Z}_{c}}=\frac{1}{2} P P_{S} \otimes$ $Q_{\hat{z} \hat{x}} Q_{\hat{x} \hat{x}}^{-1} Q_{\hat{x} \hat{z}}$. The impact this precision improvement has on ambiguity resolution will be shown in Sect. 4.

The contribution of the affine constraints to the estimators $\hat{X}_{c}$ (cf. 28) and $\hat{Z}_{c}$ (cf. 31), is felt through the presence of the projector $P_{S}$. Since a projector is invariant for the choice of basis matrix of its range, matrix $P_{S}$ remains unchanged if basis matrix $S$ is replaced by any other basis matrix of the null space of $B$. Thus, if $\tilde{S}=S U$, then $P_{S}=S\left(S^{\mathrm{T}} P S\right)^{-1} S^{\mathrm{T}} P=$ $\tilde{S}\left(\tilde{S}^{\mathrm{T}} P \tilde{S}\right)^{-1} \tilde{S}^{\mathrm{T}} P$, for any invertible matrix $U$ of order $(r-$ $q) \times(r-q)$. Similarly we have, if $\tilde{B}=V B$, then $P_{S}^{\perp}=$ $P^{-1} B^{\mathrm{T}}\left(B P^{-1} B^{\mathrm{T}}\right)^{-1} B=P^{-1} \tilde{B}^{\mathrm{T}}\left(\tilde{B} P^{-1} \tilde{B}^{\mathrm{T}}\right)^{-1} \tilde{B}$, for any invertible matrix $V$ of order $q \times q$. Hence, since $\hat{Z}_{c}$ remains 
unchanged if we replace $B$ by $V B$ for any regular transformation $V$, the constrained float ambiguity solution is not affected by a change of body-frame. This implies, since the constrained float solution is the input for ambiguity resolution, that the ambiguity success-rate is invariant for a change of body-frame. But such a change does affect, of course, the solution for the affine-attitude matrix (cf. 27).

\subsection{Unconstrained and constrained fixed baseline matrix}

Now we consider the case that $Z$ is known, referred to as the fixed solution. The corresponding unconstrained and constrained LS-solutions are denoted as $\hat{X}(Z)$ and $\hat{R}_{c}(Z)$, respectively. Their dependence on the value taken for $Z$ is explicitly shown in their argument. The $Z$-fixed LSsolution of model (22), together with its variance matrix $Q_{\hat{R}_{c}(Z) \hat{R}_{c}(Z)}=\mathrm{D}\left(\operatorname{vec}\left(\hat{R}_{c}(Z)\right)\right)$, follow from solving the system of normal equations

$$
\begin{aligned}
& {\left[B P^{-1} B^{\mathrm{T}} \otimes M^{\mathrm{T}} Q^{-1} M\right] \operatorname{vec}\left(\hat{R}_{c}(Z)\right)} \\
& \quad=\left[B P^{-1} \otimes M^{\mathrm{T}} Q^{-1}\right] \operatorname{vec}(Y-N Z)
\end{aligned}
$$

as

$$
\begin{aligned}
& \hat{R}_{c}(Z)=M^{-}[Y-N Z] B^{-} \\
& Q_{\hat{R}_{c}(Z) \hat{R}_{c}(Z)}=\left(B P^{-1} B^{\mathrm{T}}\right)^{-1} \otimes\left(M^{\mathrm{T}} Q^{-1} M\right)^{-1}
\end{aligned}
$$

with left-inverse $M^{-}=\left(M^{\mathrm{T}} Q^{-1} M\right)^{-1} M^{\mathrm{T}} Q^{-1}$ and rightinverse $B^{-}=P^{-1} B^{\mathrm{T}}\left(B P^{-1} B^{\mathrm{T}}\right)^{-1}$. Again the unconstrained solution is obtained by setting $B=I$. In doing so, we find that $\hat{X}(Z)$ and $\hat{R}_{c}(Z)$ are given as

$\hat{X}(Z)=M^{-}[Y-N Z]$ and $\hat{R}_{c}(Z)=\hat{X}(Z) B^{-}$

with variance matrices

$$
\begin{aligned}
& Q_{\hat{X}(Z) \hat{X}(Z)}=\frac{1}{2} P \otimes Q_{\hat{x}(z) \hat{x}(z)} \\
& Q_{\hat{R}_{c}(Z) \hat{R}_{c}(Z)}=\frac{1}{2}\left(B P^{-1} B^{\mathrm{T}}\right)^{-1} \otimes Q_{\hat{x}(z) \hat{x}(z)}
\end{aligned}
$$

where $Q_{\hat{x}(z) \hat{x}(z)}=2\left(M^{\mathrm{T}} Q^{-1} M\right)^{-1}$ is the variance matrix of a fixed single-baseline solution (again assuming its two antennas of equal quality). In analogy with (28), we have for $\hat{X}_{c}(Z)=\hat{R}_{c}(Z) B$,

$$
\hat{X}_{c}(Z)=\hat{X}(Z) P_{S}^{\perp}, Q_{\hat{X}_{c}(Z) \hat{X}_{c}(Z)}=\frac{1}{2} P P_{S}^{\perp} \otimes Q_{\hat{x}(z) \hat{x}(z)}
$$

Since $Z$ is assumed known, the dispersion of $\hat{X}_{c}(Z)$ (or $\hat{X}(Z)$ ) is, of course, better than that of $\hat{X}_{c}$ (or $\hat{X}$ ). Importantly, in case of GNSS, this difference is very significant. In case of GNSS, the dispersion of $\hat{X}_{c}(Z)$ is driven by the very precise carrier-phase measurements, while the dispersion of $\hat{X}_{c}$ is driven by the relatively low precision code measurements. Denoting the phase variance as $\sigma_{\phi}^{2}$ and the code variance as $\sigma_{p}^{2}$, the variance matrices of the two estimators can shown to be related as (Teunissen 1997)
$Q_{\hat{X}_{c}(Z) \hat{X}_{c}(Z)} \approx \frac{\sigma_{\phi}^{2}}{\sigma_{p}^{2}} Q_{\hat{X}_{c} \hat{X}_{c}}$

where, in case of current GPS, $\sigma_{\phi}^{2} / \sigma_{p}^{2} \approx 10^{-4}$. This shows, if one would be able to determine $Z$ with negligible uncertainty, that a very large precision improvement in the determinations of the array-baseline matrix $X$ and the affine-attitude matrix $R$ can be realized. Determining $Z$ with such negligible uncertainty is the goal of integer ambiguity resolution.

\subsection{Unconstrained and constrained integer least-squares solution}

With the expressions of the 'float' and 'fixed' baseline and ambiguity matrix solutions given, we are now in a position to derive the corresponding unconstrained and constrained integer least-squares solutions.

\subsubsection{Unconstrained integer least-squares}

We start with the unconstrained ILS solution

$$
\begin{aligned}
{\left[\check{X}^{\mathrm{T}}, \check{Z}^{\mathrm{T}}\right]^{\mathrm{T}}=} & \underset{X \in \mathbb{R}^{3 \times r}, Z \in \mathbb{Z}^{f s \times r}}{\arg \min } \\
& \times\left\|\operatorname{vec}\left(Y-[M, N]\left[X^{\mathrm{T}}, Z^{\mathrm{T}}\right]^{\mathrm{T}}\right)\right\|_{Q_{Y Y}}^{2}
\end{aligned}
$$

In order to show how this solution can be determined from the unconstrained 'float' solutions, use is made of the following lemma.

Lemma 2 (Multivariate Orthogonal Decomposition) Let $\hat{X}, \hat{Z}, \hat{X}(Z)$ and their variance matrices be given as in (27), (29) and (34), and let $\hat{E}=Y-M \hat{X}-N \hat{Z}$. Then

$$
\begin{aligned}
& \|\operatorname{vec}(Y-M X-N Z)\|_{Q_{Y Y}}^{2}=\|\operatorname{vec}(\hat{E})\|_{Q_{Y Y}}^{2} \\
& \quad+\|\operatorname{vec}(\hat{Z}-Z)\|_{Q_{\hat{Z} \hat{Z}}}^{2}+\|\operatorname{vec}(\hat{X}(Z)-X)\|_{Q_{\hat{X}(Z) \hat{X}(Z)}}^{2}
\end{aligned}
$$

Proof The proof is given in the Appendix.

Expression (38) decomposes the multivariate objective function into three terms, the first of which is a constant, the second of which only varies with $Z$, and the third of which varies with both $X$ and $Z$. With the aid of this decomposition, the minimization of the objective function can be decomposed as well, namely as

$$
\begin{aligned}
& \min _{X \in \mathbb{R}^{3 \times r}, Z \in \mathbb{Z}^{f s \times r}}\|\operatorname{vec}(Y-M X-N Z)\|_{Q_{Y Y}}^{2} \\
= & \|\operatorname{vec}(\hat{E})\|_{Q_{Y Y}}^{2}+\min _{Z \in \mathbb{Z}^{f s \times r}}\left(\|\operatorname{vec}(\hat{Z}-Z)\|_{Q_{\hat{Z} \hat{Z}}^{2}}\right. \\
& \left.+\min _{X \in \mathbb{R}^{3 \times r}}\|\operatorname{vec}(\hat{X}(Z)-X)\|_{Q_{\hat{X}(Z) \hat{X}(Z)}^{2}}^{2}\right)
\end{aligned}
$$

Since the third term on the right-hand side can be made zero for any $Z$, the unconstrained ILS-estimators $\breve{Z}$ and $\breve{X}$ follow as

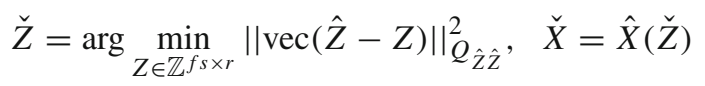


This multivariate ILS problem can be solved by standard means, since the ambiguity objective function is quadratic in $\operatorname{vec}(Z)$ (Teunissen 1995). Note, if the baseline matrix would be required to satisfy the matrix constraint $X^{\mathrm{T}} X=C$ as in (14), that the third term on the right-hand side of (39) would not vanish and the ambiguity objective function would be given by (16), instead of by the quadratic one of (40).

\subsubsection{Constrained integer least-squares}

To determine the constrained ILS estimators, $\check{Z}_{c}$ and $\check{X}_{c}$ (cf. 23), we substitute $X=R B$ into (39) and minimize over $R \in \mathbb{R}^{3 \times q}$ instead of over $X \in \mathbb{R}^{3 \times r}$. This gives

$$
\begin{aligned}
& \min _{R \in \mathbb{R}^{3 \times q}, Z \in \mathbb{Z}^{f s \times r}}\|\operatorname{vec}(Y-M R B-N Z)\|_{Q_{Y Y}}^{2} \\
& =\|\operatorname{vec}(\hat{E})\|_{Q_{Y Y}}^{2}+\min _{Z \in \mathbb{Z}^{f s \times r}}\left(\|\operatorname{vec}(\hat{Z}-Z)\|_{Q_{\hat{Z} \hat{Z}}^{2}}^{2}\right. \\
& \left.\quad+\min _{R \in \mathbb{R}^{3 \times q}}\|\operatorname{vec}(\hat{X}(Z)-R B)\|_{Q_{\hat{X}(Z) \hat{X}(Z)}}^{2}\right)
\end{aligned}
$$

Now, in contrast to (39), the third term on the right-hand side does not vanish anymore. This term can be further decomposed, in analogy with (38), as

$$
\begin{aligned}
& \|\operatorname{vec}(\hat{X}(Z)-R B)\|_{Q_{\hat{X}(Z) \hat{X}(Z)}}^{2}=\left\|\operatorname{vec}\left(\hat{X}(Z) P_{S}\right)\right\|_{Q_{\hat{X}(Z) \hat{X}(Z)}^{2}}^{2} \\
& \quad+\left\|\operatorname{vec}\left(\hat{R}_{c}(Z)-R\right)\right\|_{Q_{\hat{R}_{c}(Z) \hat{X}_{c}(Z)}^{2}}^{2}
\end{aligned}
$$

with residual matrix $\hat{X}(Z) P_{S}=\hat{X}(Z)-\hat{R}_{c}(Z) B$ (cf. 35). From substituting (42) into (41), it follows that the constrained ILS solution is given as

$$
\check{Z}_{c}=\arg \min _{Z \in \mathbb{Z}^{f s \times r}} J(Z), \quad \check{X}_{c}=\hat{R}_{c}\left(\check{Z}_{c}\right) B
$$

with the ambiguity objective function given as

$$
J(Z)=\|\operatorname{vec}(\hat{Z}-Z)\|_{Q_{\hat{Z} \hat{Z}}}^{2}+\left\|\operatorname{vec}\left(\hat{X}(Z) P_{S}\right)\right\|_{Q_{\hat{X}(Z) \hat{X}(Z)}}^{2}
$$

Compare this constrained ILS solution with its unconstrained counterpart (40). Note, importantly, that the ambiguity objective function of $\breve{Z}_{c}$ differs from that of $\breve{Z}$ by the presence of the $P_{S}$-dependent second term in (44). The presence of both constraints, the integer-constraint and the affine-constraint, is therefore felt when evaluating this objective function. In integer minimizing $J(Z)$ not only the weighted distance between $Z$ and $\hat{Z}$ counts (as is the case in 40), but also by how much $\hat{X}(Z)$ violates the affine-constraint $X S=0$. Two types of weights are involved, the inverse of $Q_{\hat{Z} \hat{Z}}$ and the inverse of $Q_{\hat{X}(Z) \hat{X}(Z)}$. As remarked earlier, in case of GNSS, the variance matrix of $\hat{X}(Z)$ is driven by the very precise carrierphase data, while that of $\hat{Z}$ is driven by the relatively poor precision code data. Hence, the second term in the ambiguity objective function (44) receives a relatively large weight and contributes therefore, as shown in Sect. 4, significantly to the improved success rate performance of $\breve{Z}_{c}$ over $\check{Z}$.

\subsubsection{The integer search}

Just like the unconstrained ILS problem (40), also the constrained ILS problem (43) can be solved using standard methods. This is the advantage the affine-constrained ambiguity objective function $J(Z)$ of (43) has over the nonquadratic ambiguity objective function $H(Z)$ of (16). We illustrate this by showing how the two quadratic terms of $J(Z)$ can be combined into one single quadratic form. For that purpose, we make use of a different orthogonal decomposition then the one used in (41). Instead of using the unconstrained 'float' and 'fixed' solutions, $\hat{Z}$ and $\hat{X}(Z)$, one can also use the constrained 'float' and 'fixed' solutions, $\hat{Z}_{c}$ and $\hat{R}_{c}(Z)$, to decompose the objective function. We therefore have, in analogy of (38), the orthogonal decomposition

$$
\begin{aligned}
& \|\operatorname{vec}(Y-M R B-N Z)\|_{Q_{Y Y}}^{2}=\left\|\operatorname{vec}\left(\hat{E}_{c}\right)\right\|_{Q_{Y Y}}^{2} \\
& \quad+\left\|\operatorname{vec}\left(\hat{Z}_{c}-Z\right)\right\|_{Q_{\hat{Z}_{c} \hat{Z}_{c}}}^{2}+\left\|\operatorname{vec}\left(\hat{R}_{c}(Z)-R\right)\right\|_{Q_{\hat{R}_{c}(Z) \hat{R}_{c}(Z)}}^{2}
\end{aligned}
$$

in which $\hat{E}_{c}=Y-M \hat{R}_{c} B-N \hat{Z}_{c}$ is the affine-constrained LS-residual matrix. From equating this alternative decomposition to the original one (38), it follows that

$$
\begin{aligned}
\|\operatorname{vec}(\hat{E})\|_{Q_{Y Y}}^{2}+J(Z)= & \left\|\operatorname{vec}\left(\hat{E}_{c}\right)\right\|_{Q_{Y Y}}^{2} \\
& +\left\|\operatorname{vec}\left(\hat{Z}_{c}-Z\right)\right\|_{Q_{\hat{Z}_{c} \hat{z}_{c}}}^{2}
\end{aligned}
$$

This shows that the integer minimizer $\check{Z}_{c}$ of $J(Z)$ can indeed be found by means of integer minimizing a single quadratic form as

$$
\check{Z}_{c}=\arg \min _{Z \in \mathbb{Z}^{f s \times r}}\left\|\operatorname{vec}\left(\hat{Z}_{c}-Z\right)\right\|_{Q_{\hat{Z}_{c} \hat{z}_{c}}}^{2}
$$

Hence, just like in the unconstrained case (40), the affine-constrained ILS ambiguity matrix $\breve{Z}_{c}$ can be computed efficiently by standard means.

\subsubsection{Multi-epoch solution}

The solutions given so far are single-epoch solutions. They therefore apply to instantaneous ambiguity resolution. In case of multiple epochs, the inclusion of time in the affine-constrained GNSS attitude model (22) leads to the formulation

$\mathrm{E}(Y(t))=M(t) X(t)+N Z, X(t)=R(t) B$

$\mathrm{D}(\operatorname{vec}(Y(t)))=P \otimes Q(t)$

in which the time dependence is made explicit through the use of the argument $t$. In (48) the time-invariant variates are: the ambiguity matrix $Z$, its coefficient matrix $N$, the body-frame 
array geometry $B$, and the relative quality of the array antennas $P$. When solving (48) for multiple epochs, one obtains, in analogy with (31), a multi-epoch, affine-constrained float ambiguity matrix solution, say $\hat{Z}_{c}(t)$, with variance matrix $Q_{\hat{Z}_{c}(t) \hat{Z}_{c}(t)}$. This solution will be more precise than the single-epoch solutions treated so far, since it will have profitted from the assumed ambiguity time-invariance in (48). The corresponding ILS solution follows from replacing $\hat{Z}_{c}$ and $Q_{\hat{Z}_{c} \hat{Z}_{c}}$, in (47), by $\hat{Z}_{c}(t)$ and $Q_{\hat{Z}_{c}(t) \hat{Z}_{c}(t)}$, respectively. Hence, the multi-epoch, affine-constrained ILS ambiguity matrix can also be computed by standard means.

Since the ambiguities are the only parameters in (48), that are assumed linked in time, the model becomes decoupled in time once the integer ambiguity matrix is resolved. Hence, once the ambiguity matrix can be assumed known, the ambiguity-resolved solutions for the attitude matrix $R(t)$ can be produced again on an epoch-by-epoch basis.

\section{The affine constrained ambiguity dilution of precision}

In this section, the ambiguity dilution of precision (ADOP) of the affine-constrained GNSS attitude model is described and analysed. A closed-form expression for it is derived, thus providing a very simple and insightful rule-of-thumb describing the model's instantaneous ambiguity resolution capabilities.

\subsection{ADOP: definition and properties}

The ADOP was introduced in (Teunissen 1997) as an easyto-compute scalar diagnostic to measure the intrinsic model strength for successful ambiguity resolution. The ADOP is defined as the square-root of the ambiguity variance matrix' determinant taken to the power one over the number of DD ambiguities. Thus if $Q_{\hat{a} \hat{a}}$ would be the $n \times n$ ambiguity variance matrix, then its ADOP would read

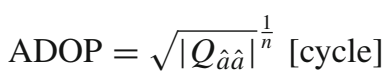

No actual measurements are needed to compute the ADOP, only the ambiguity variance matrix. Hence, the ADOP can be used as a design parameter (Teunissen and Odijk 1997). In the following, we will analyse the ADOPs for $\hat{a}=\operatorname{vec}(\hat{Z})$ and $\hat{a}=\operatorname{vec}\left(\hat{Z}_{c}\right)$, respectively.

The ADOP has been used in (Skaloud 1998) and (Scherzinger 2000, 2001) to analyse the benefits to ambiguity resolution of integrating INS with GPS, while in (Lee et al. 2010), it has been used to investigate the combined effect of GPS, INS and pseudolite integration. In (Vollath et al. 2003), the ADOP was used to study the impact of the number of GPS and Galileo carriers on ambiguity resolution, while in (Jonkman and Teunissen 2001) it was used to analyse geometry-free full ambiguity resolution for long baselines. In
(Wang et al. 2005), the ADOP was used to study the effect the stochastic model has on ambiguity resolution, while in (Ong 2010) it was used to quantify the contribution to ambiguity resolution of combining GPS with GLONASS. To study the ambiguity resolution potential of low-cost, multi-GNSS receiver data, the ADOP was used by Verhagen et al. (2010) to capture the capabilities of single-frequency, GPS-Galileo integration.

Note that the above ambiguity-DOP definition differs from the position-related DOP measures, such as the position (PDOP), the vertical (VDOP) or the horizontal dilution of precision (HDOP), see e.g. (Hofmann-Wellenhof et al. 2008; Leick 2004; Strang and Borre 1997). These latter DOP measures are all based on the trace of the variance matrix of the coordinates, instead of on its determinant. In case of ambiguities, however, the trace should not be used. First, the trace of an ambiguity variance matrix is not invariant under ambiguity transformations. Second, the trace does not take the correlation between ambiguities into account, while it is known that the DD ambiguities can be highly correlated, especially in case of short observation times.

The ADOP has the important property that it is invariant against the choice of ambiguity parametrization. Since all admissible ambiguity transformations can be shown to have a determinant of one, the ADOP does not change when one changes the definition of the ambiguities. It therefore measures the intrinsic precision of the ambiguities. In fact, it measures the average ambiguity precision, since the ADOP equals the geometric average of the sequential conditional ambiguity standard deviations.

Another important property of the ADOP is its relation to the volume of the ambiguity confidence ellipsoid and the ambiguity search space. The volume of the $n$-dimensional ellipsoid $(\hat{a}-a)^{T} Q_{\hat{a} \hat{a}}^{-1}(\hat{a}-a) \leq \chi^{2}$ is given as $V_{n}=\chi^{n} U_{n} \mathrm{ADOP}^{n}$ where $U_{n}$ is the volume of the $n$-dimensional unit sphere (Teunissen et al. 1996). Thus if the ADOP gets smaller, the confidence ellipsoid gets smaller and the multivariate PDF gets more peaked.

The ADOP can also be directly linked to the probability of correct integer estimation, the ambiguity success-rate (Teunissen 1998). Since the ADOP is a measure for the average ambiguity precision, the probability

$P_{\mathrm{ADOP}}=\left[2 \Phi\left(\frac{1}{2 A D O P}\right)-1\right]^{n}$

with $\Phi(x)=\int_{-\infty}^{x} \frac{1}{\sqrt{2 \pi}} \exp \left\{-\frac{1}{2} v^{2}\right\} d v$, has been shown to give a good approximation to the success-rate (Verhagen 2005; Ji et al. 2007). Figure 1 shows the $P_{\mathrm{ADOP}}$ as function of ADOP for varying levels of $n(n=1, \ldots, 60)$. It can be seen that the ADOP-based success rate decreases for increasing ADOP and this decrease is steeper the more ambiguities are involved. In general, Fig. 1 shows that if ADOP is smaller than about 0.10 cycle, $P_{\text {ADOP }}$ becomes larger than 


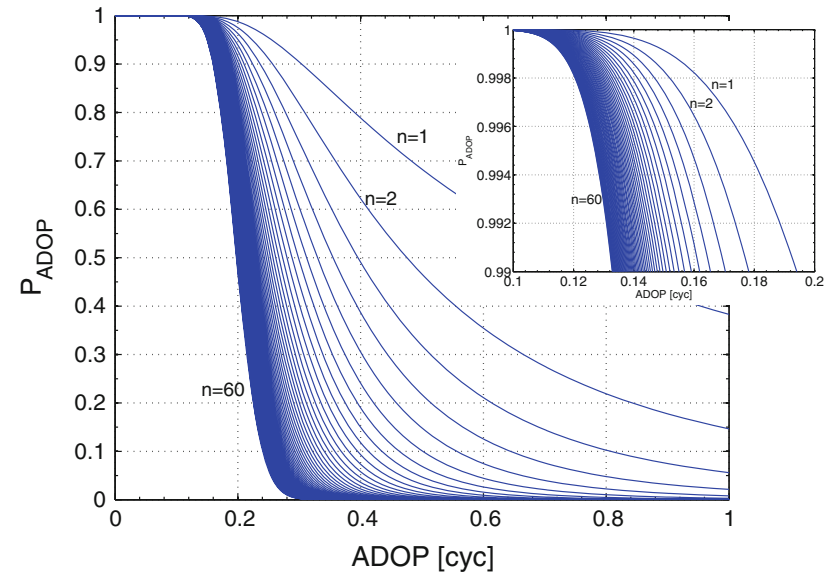

Fig. $1 P_{\mathrm{ADOP}}$ versus $\mathrm{ADOP}$ for varying number of DD ambiguities $1 \leq n \leq 60$

0.999 , while for ADOP smaller than 0.13 cycle, $P_{\text {ADOP }}$ is always better than 0.99 .

\subsection{The effect of the affine-constraints}

The affine-constraints of the GNSS attitude model increases the model's capabilities of successful integer ambiguity resolution. To measure the effect these affine-constraints have on the ADOP, we first need a general rule describing how the ADOP is impacted by the presence of linear constraints. The following theorem describes the effect arbitrary linear constraints have on the ADOP.

Theorem 1 (The Constrained ADOP) Consider the linear model $\mathrm{E}(y)=A a+B b, \mathrm{D}(y)=Q_{y y}$. Let $\check{c}$ and $\hat{c}$, with variance matrices $Q_{\breve{c} \check{c}}$ and $Q_{\hat{c} \hat{c}}$, be the LS-estimators of the linear functions $c=C b$, assuming the n-vector a known and unknown, respectively. Furthermore, let $\mathrm{ADOP}_{\mathrm{C}}$ and $\mathrm{ADOP}$ be the ambiguity-dilution-of-precision of the LS-estimators of $a$, assuming c known and unknown, respectively. Then

$\mathrm{ADOP}_{\mathrm{C}}=\sqrt{\frac{\left|Q_{\breve{c} \check{c}}\right|}{\left|Q_{\hat{c} \hat{c}}\right|}} \mathrm{ADOP}^{\frac{1}{n}} \mathrm{ADO}$

Proof The proof is given in the Appendix.

This result shows that the effect of the constraint $c=C b$ on the ADOP is driven by the ratio $\left|Q_{\breve{c} \check{c}}\right| /\left|Q_{\hat{c} \hat{c}}\right|$ and thus by the gain in precision of estimating $c$ when knowing $a$. Thus the effect these constraints have on the ADOP is driven by how much better these constraints can be estimated once the ambiguities are known. If knowledge of $a$ improves our ability of estimating $c=C b$, then so will knowledge of $c$ improve the ADOP. However, if the LS-estimators $\hat{a}$ and $\hat{c}=C \hat{b}$ would be uncorrelated, then constraining $c=C b$ would do nothing for improving the ADOP.
We now apply the above theorem to the affine-constrained GNSS attitude model (20). The affine matrix constraint $X S=$ 0 reads in matrix-vector form $\left(S^{\mathrm{T}} \otimes I_{r}\right) \operatorname{vec}(X)=0$. Hence, in order to apply the above theorem, we need the variance matrix of $\left(S^{\mathrm{T}} \otimes I_{r}\right) \operatorname{vec}(X)$ for the two cases, $X=\hat{X}$ and $X=\hat{X}(Z)$, respectively. With (27) and (34), these two required variance

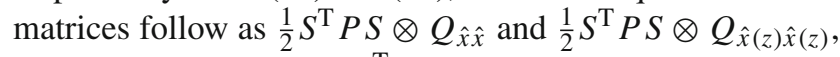
respectively. The matrix $S^{\mathrm{T}} P S$ is of order $(r-q) \times(r-q)$. We therefore have

$\frac{\left|Q_{\breve{c} \breve{c}}\right|}{\left|Q_{\hat{c} \hat{c}}\right|}:=\frac{\left|\frac{1}{2} S^{\mathrm{T}} P S \otimes Q_{\hat{x}(z) \hat{x}(z)}\right|}{\left|\frac{1}{2} S^{\mathrm{T}} P S \otimes Q_{\hat{x} \hat{x}}\right|}=\left(\frac{\left|Q_{\hat{x}(z) \hat{x}(z)}\right|}{\left|Q_{\hat{x} \hat{x}}\right|}\right)^{r-q}$

where use is made of the Kronecker product property $\mid A \otimes$ $\left.B|=| A\right|^{b}|B|^{a}$ for any $a \times a$ matrix $A$ and any $b \times b$ matrix $B$. Since in our case the number of ambiguities is $n=f s r$, the following lemma follows from combining (51) and (52).

Lemma 3 (Impact of Affine Constraints) Let the affineconstrained and unconstrained ADOP of the GNSS attitude model (20) be given as $\mathrm{ADOP}_{\mathrm{AC}}=\left|Q_{\hat{Z}_{c} \hat{Z}_{c}}\right|^{\frac{1}{2 f s r}}$ and $\mathrm{ADOP}_{\mathrm{UC}}=\left|Q_{\hat{Z} \hat{Z}}\right|^{\frac{1}{2 f s r}}$, respectively. Then, for $r \geq q$,

$\operatorname{ADOP}_{\mathrm{AC}}=\left(\sqrt{\frac{\left|Q_{\hat{x}(z) \hat{x}(z)}\right|}{\left|Q_{\hat{x} \hat{x}}\right|}}\right)^{\frac{1}{f s}\left(1-\frac{q}{r}\right)} \mathrm{ADOP}_{\mathrm{UC}}$

Note that $\mathrm{ADOP}_{\mathrm{AC}}=\mathrm{ADOP}_{\mathrm{UC}}$ when $r=q$. This is the case, of course, when there are no affine-constraints, i.e. when the number of baselines equals the dimension of their span. From (53) it follows that the affine constraints have more impact if the ratio $\left|Q_{\hat{x}(z) \hat{x}(z)}\right| /\left|Q_{\hat{x} \hat{x}}\right|$ is smaller. Thus it is the gain in baseline precision due to ambiguity resolution that drives the ADOP improvement. If there is no such gain, i.e. $\left|Q_{\hat{x}(z) \hat{x}(z)}\right| /\left|Q_{\hat{x} \hat{x}}\right|=1$, then also no benefits will follow from imposing the affine-constraints.

Table 1 shows the factor by which the ADOP improves for a typical single-frequency scenario. It gives the ratio $\mathrm{ADOP}_{\mathrm{AC}} / \mathrm{ADOP}_{\mathrm{UC}}$ as function of the number of antennas and satellites, assuming a phase-code variance ratio of $\sigma_{\phi}^{2} / \sigma_{p}^{2}=10^{-4}$. Note that the impact of the affine constraints can be very significant indeed. Also note that the benefit of

Table 1 The ratio $\mathrm{ADOP}_{\mathrm{AC}} / \mathrm{ADOP}_{\mathrm{UC}}$, for a single-frequency array $(f=1)$, having a three-dimensional span $(q=3)$ and phase-code variance ratio $\sigma_{\phi}^{2} / \sigma_{p}^{2}=10^{-4}$, as function of the number of antennas $(r+1)$ and the number of tracked satellites $(s+1)$

\begin{tabular}{lllll}
\hline & $r=3$ & $r=4$ & $r=5$ & $r=6$ \\
\hline$s=3$ & 1 & .32 & .16 & .10 \\
$s=4$ & 1 & .42 & .25 & .18 \\
$s=5$ & 1 & .50 & .33 & .25 \\
$s=6$ & 1 & .56 & .40 & .32 \\
\hline
\end{tabular}


having more antennas is felt more when fewer satellites are tracked. This is an important property as it shows that the improvement is targeted at where it is needed. The unconstrained ADOP will namely already improve significantly when the number of tracked satellites increases.

\subsection{The affine-constrained ADOP in closed form}

We now present an easy-to-use expression for the affineconstrained ADOP. It will exemplify the role of the various contributing factors, such as phase- and code-precision, number of frequencies and their wavelengths, and number of tracking antennas and tracked satellites. From it the expected instantaneous ambiguity resolution performances of one-dimensional, two-dimensional and three-dimensional antenna arrays can be inferred. We have the following general result.

Theorem 2 (The Affine Constrained ADOP) The ambiguity-dilution-of-precision of the affine-constrained GNSS attitude model (20) is given as

$\operatorname{ADOP}_{\mathrm{AC}}=\frac{v_{\phi}}{\bar{\lambda}}\left(\sqrt{\frac{\left|Q_{\hat{x} \hat{x}}\right|}{\left|Q_{\hat{x}(z) \hat{x}(z)}\right|}}\right)^{\frac{q}{f s r}} \quad(r \geq q)$

with $v_{\phi}^{2}=\left|D_{r}^{\mathrm{T}} Q_{r} D_{r}\right|^{\frac{1}{r}}\left|Q_{f}\right|^{\frac{1}{f}}\left|D_{s}^{\mathrm{T}} Q_{\phi} D_{s}\right|^{\frac{1}{s}}$ and $\bar{\lambda}=\left(\prod_{i=1}^{f}\right.$ $\left.\lambda_{i}\right)^{\frac{1}{f}}$.

The result (54) can be further simplified as follows. If the co-factor matrices of the undifferenced phase and code data are scaled versions of each other, $Q_{\phi}=\epsilon^{2} Q_{p}$, then $\left|Q_{\hat{x} \hat{x}}\right| /\left|Q_{\hat{x}(z) \hat{x}(z)}\right|=\left(1+1 / \epsilon^{2}\right)^{3}$. And if the co-factor matrices $Q_{r}, Q_{f}$ and $Q_{\phi}$ are diagonal, with entries defined as $Q_{r}^{-1}=\operatorname{diag}\left[r_{1}, \ldots, r_{r+1}\right], Q_{f}=\operatorname{diag}\left[f_{1}, \ldots, f_{f}\right]$ and $\sigma_{\phi}^{2} Q_{\phi}^{-1}=\operatorname{diag}\left[s_{1}, \ldots, s_{s+1}\right]$, then $\left|Q_{f}\right|^{\frac{1}{f}}=\left[\prod_{i=1}^{f} f_{i}\right]^{\frac{1}{f}}$, $\left|D_{r}^{\mathrm{T}} Q_{r} D_{r}\right|^{\frac{1}{r}}=\left[\left(\sum_{i=1}^{r+1} r_{i}\right) /\left(\prod_{i=1}^{r+1} r_{i}\right)\right]^{\frac{1}{r}}$ and $\left|D_{s}^{\mathrm{T}} Q_{\phi} D_{s}\right|^{\frac{1}{s}}=$ $\sigma_{\phi}^{2}\left[\left(\sum_{i=1}^{s+1} s_{i}\right) /\left(\prod_{i=1}^{s+1} s_{i}\right)\right]^{\frac{1}{s}}$.

Proof The proof is given in the Appendix.

Expression (54) can be used to determine other ADOPs as well. In particular consider the two extremes of knowing the complete array geometry in the reference frame versus the unconstrained case of not knowing that geometry at all. In the first case, $Q_{\hat{x} \hat{x}}=0$ and the ADOP reduces to $v_{\phi} / \bar{\lambda}$. The other extreme of having no constraints corresponds with $r=q$. In that case, expression (54) becomes the ADOP of an unconstrained array or small multi-frequency GNSS network. And it reduces to that of an unconstrained, multifrequency, single-baseline if $r=q=1$.

Note that in the unconstrained case the dependence of the ADOP on $r$, i.e. the number of baselines, is only felt, via $v_{\phi}$, through $\left|D_{r}^{\mathrm{T}} Q_{r} D_{r}\right|^{\frac{1}{2 r}}$. This dependence can be shown to be rather weak however. If we assume all antennas to be of the same quality and therefore take $Q_{r}=I_{r+1}$, then

$\left|D_{r}^{\mathrm{T}} Q_{r} D_{r}\right|^{\frac{1}{2 r}}=\sqrt{r+1}^{\frac{1}{r}} \in(1, \sqrt{2}]$

This shows that in the unconstrained case, one can not drive the ADOP to much smaller values by working with much larger $r$. The factor by which the ADOP can be made smaller is at most only $\sqrt{2}$. This is in stark contrast with the effect the number of satellites has on the unconstrained ADOP (cf. 54). This is why it is so relevant that in the constrained case the improvement in the ADOP, for larger $r$, is particularly happening when fewer satellites are tracked (cf. Table 1).

Corollary (ADOP rule-of-thumb) If $Q_{r}=I_{r+1}, Q_{f}=$ $I_{f}, Q_{\phi}=\sigma_{\phi}^{2} I_{s+1}$ and $Q_{\phi}=\epsilon^{2} Q_{p}$, then the affine-constrained ADOP simplifies to

$\mathrm{ADOP}_{\mathrm{AC}}=\frac{\sigma_{\phi}}{\bar{\lambda}} \sqrt{(r+1)^{\frac{1}{r}}(s+1)^{\frac{1}{s}}}\left(\sqrt{1+\frac{\sigma_{p}^{2}}{\sigma_{\phi}^{2}}}\right)^{\frac{3 q}{f s r}}$

for $r \geq q$.

Proof The proof follows directly from Theorem 2.

This very useful and insightful rule-of-thumb clearly shows how the affine-constrained ADOP is made up from the contributing factors for successful ambiguity resolution. The contributing factors are:

- the undifferenced standard deviations: $\sigma_{\phi}, \sigma_{p}$

- the geometric average of wavelengths: $\bar{\lambda}$

- the number of frequencies: $f$

- the number of receivers/antennas: $r+1$

- the number of tracked satellites: $s+1$

- the dimension of the baseline span: $q$

The ADOP gets smaller for larger $f, s$ or $r$. The significant contribution of the affine-constraints is that one can now 'trade' satellites for receiver/antennas. This is due to the symmetry in $s$ and $r$ of (56). Such symmetry is absent in the unconstrained case. As (56) shows, the contribution of the code data precision is relative to that of the phase data. This shows that one can improve the ADOP considerably by improving the code data precision. In case of current GPS, however, the code-phase variance ratio is large, $\sigma_{p}^{2} / \sigma_{\phi}^{2}=10^{+4}$, thus requiring the product $f s r$ to be large enough so as to dampen the detoriating effect of the relatively poor code precision. With future GNSSs, however, the code precision is expected to be better, thus permitting smaller values for $f s r$.

Expression (56) also shows the effect of $q$, the dimension of the baseline span. The smaller $q$ is, the more constrained the model is and thus the smaller the ADOP is. Full attitude 
Table $2 \mathrm{ADOP}_{\mathrm{AC}}$ as function of number of satellites $(s+1)$ and number of receiver/antennas $(r+1)$, for a single-frequency $(f=1)$ threedimensional baseline span $(q=3)$, with $\sigma_{\phi} / \bar{\lambda}=0.01$ and $\sigma_{\phi} / \sigma_{p}=$ 0.01

\begin{tabular}{lrccc}
\hline ADOP $_{\text {AC }}$ [cycle] & $r=3$ & $r=4$ & $r=5$ & $r=6$ \\
\hline$s=3$ & 1.59 & .49 & .24 & .15 \\
$s=4$ & .49 & .20 & .12 & .08 \\
$s=5$ & .24 & .12 & .08 & .06 \\
$s=6$ & .15 & .08 & .06 & .04 \\
\hline
\end{tabular}

Table 3 ADOP $_{\text {AC }}$ as function of number of satellites $(s+1)$ and number of receiver/antennas $(r+1)$, for a single-frequency $(f=1)$ two-dimensional baseline span $(q=2)$, with $\sigma_{\phi} / \bar{\lambda}=0.01$ and $\sigma_{\phi} / \sigma_{p}=0.01$

\begin{tabular}{lcccc}
\hline ADOP $_{\text {AC }}$ [cycle] & $r=2$ & $r=3$ & $r=4$ & $r=5$ \\
\hline$s=3$ & 1.66 & .34 & .15 & .10 \\
$s=4$ & .51 & .15 & .09 & .06 \\
$s=5$ & .25 & .10 & .06 & .04 \\
$s=6$ & .15 & .06 & .05 & .04 \\
\hline
\end{tabular}

Table 4 ADOP $_{\text {AC }}$ as function of number of satellites $(s+1)$ and number of receiver/antennas $(r+1)$, for a single-frequency $(f=1)$ one-dimensional baseline span $(q=1)$, with $\sigma_{\phi} / \bar{\lambda}=0.01$ and $\sigma_{\phi} / \sigma_{p}=0.01$

\begin{tabular}{lcccc}
\hline ADOP $_{\text {AC }}$ [cycle] & $r=1$ & $r=2$ & $r=3$ & $r=4$ \\
\hline$s=3$ & 1.78 & .18 & .08 & .05 \\
$s=4$ & .55 & .09 & .05 & .04 \\
$s=5$ & .27 & .06 & .04 & .03 \\
$s=6$ & .17 & .05 & .03 & .03 \\
\hline
\end{tabular}

recovery is possible in case $q=2$ or $q=3$, while only heading and elevation can be determined in case $q=1$. To illustrate the expected ambiguity resolution performance of the affine-constrained GNSS attitude model, the values for the single-frequency $\mathrm{ADOP}_{\mathrm{AC}}$ are given in Tables 2, 3, 4 for different values of $q$. The single-frequency case is chosen as this is considered to be the most challenging case for attitude determination.

As Table 2 shows, one needs either a sufficient number of satellites or a sufficient number of receiver/antennas to get a small enough ADOP in case $q=3$. In case of three baselines $(r=3)$, one needs seven $(s=6)$ or more satellites to get a small enough ADOP. However, by adding antennas one can reduce the ADOP significantly. For instance, for $r=s=5$, one can expect successful ambiguity resolution, since then $\mathrm{ADOP}_{\mathrm{AC}}=0.08$ cycle. Note that in the unconstrained case, this is not possible, since the ADOP is then 3 times larger (cf. Table 1).

The results of Table 3 apply to the case when all antennas are situated in a plane $(q=2)$. These ADOP values are smaller than their counterparts of Table 2 . With three baselines $(r=3)$ and six satellites $(s=5)$, we already have $\mathrm{ADOP}_{\mathrm{AC}}=0.1$ cycle, from which follows that one can expect to have successful ambiguity resolution.

The results of Table 4 apply to the case when all antennas are aligned in a straight line $(q=1)$. These ADOP values are again smaller than their counterparts of Tables 2 and 3, respectively. The values in the Table show that already with three antennas $(r=2)$ and five satellites $(s=4)$, successful ambiguity resolution can be expected.

Although these instantaneous single-frequency ambiguity resolution capabilities of the affine-constrained GNSS attitude model are already impressive, they get even better when more epochs are used. Due to the relatively slow changing receiver-satellite geometry, the $k$-epoch affine-constrained $\mathrm{ADOP}$ is to a good approximation equal to $\frac{1}{\sqrt{k}} \mathrm{ADOP}_{\mathrm{AC}}$. This expression can be used in combination with the results given in the Tables to infer the multi-epoch ambiguity resolution capabilities of the affine-constrained model.

\section{Summary and conclusion}

In this contribution, the affine-constrained GNSS attitude model was introduced. This model avoids the computational complexity of the orthonormality-constrained GNSS attitude model, while it still has a better ambiguity resolution performance than the multivariate unconstrained GNSS model. By formulating the parametric orthonormality constraints of a $q$-dimensional, $(r+1)$-antenna array in an equivalent implicit form, the complete set of constraints could be decomposed into $3(r-q)$ linear constraints and $\frac{1}{2} q(q+1)$ quadratic constraints. The linear constraints are referred to as affine, since a discarding of the quadratic constraints is equivalent to assuming that the reference-frame and body-frame baseline matrices are related by an affine transformation instead of by a rotation.

It was shown that the ambiguity objective function of the affine-constrained GNSS attitude model is given by

$$
J(Z)=\|\operatorname{vec}(\hat{Z}-Z)\|_{Q_{\hat{Z} \hat{Z}}}^{2}+\left\|\operatorname{vec}\left(\hat{X}(Z) P_{S}\right)\right\|_{Q_{\hat{X}(Z) \hat{X}(Z)}}^{2}
$$

while that of the more complex orthonormality-constrained model is given by

$$
H(Z)=J(Z)+\left\|\operatorname{vec}\left(\hat{R}_{c}(Z)-\check{R}_{c}(Z)\right)\right\|_{Q_{\hat{R}_{c}(Z) \hat{R}_{c}(Z)}^{2}}^{2}
$$

with

$$
\check{R}_{c}(Z)=\arg \min _{R \in \mathbb{O}^{3 \times q}}\left\|\operatorname{vec}\left(\hat{R}_{c}(Z)-R\right)\right\|_{Q_{\hat{R}_{c}(Z) \hat{R}_{c}(Z)}^{2}}
$$

The second term on the right-hand side of (57) is due to the $3(r-q)$ linear constraints, while the second term on the righthand side of (58) is due to the $\frac{1}{2} q(q+1)$ quadratic constraints. 
The advantage of $J(Z)$ over $H(Z)$ is that $J(Z)$ is quadratic in $Z$ while $H(Z)$ is not. The search space of $J(Z)$ is therefore ellipsoidal, from which follows that its integer minimizer can be obtained efficiently by standard means. This is not the case for $H(Z)$. Its search space is non-ellipsoidal, as a consequence of which a non-standard and more complex search needs to be executed for computing the integer minimizer of $H(Z)$ (Giorgi et al. 2011).

Since the success-rate of the integer minimizer of $J(Z)$ can expected to be smaller than that of $H(Z)$, it is important to understand under which circumstances the affine-constrained GNSS attitude model will still allow successful ambiguity resolution. For this purpose a general closed form formula for its ADOP was derived, giving for $r \geq q$ the simple and insightful rule-of-thumb

$\operatorname{ADOP}_{\mathrm{AC}}=\frac{\sigma_{\phi}}{\bar{\lambda}} \sqrt{(r+1)^{\frac{1}{r}}(s+1)^{\frac{1}{s}}}\left(\sqrt{1+\frac{\sigma_{p}^{2}}{\sigma_{\phi}^{2}}}\right)^{\frac{3 q}{f s r}}$

This expression clearly shows how the affine-constrained ADOP is made up from the contributing factors for successful ambiguity resolution. These factors are the undifferenced standard deviations of phase and code, $\sigma_{\phi}$ and $\sigma_{p}$, the geometric average of wavelengths, $\bar{\lambda}$, the number of frequencies, $f$, the number of receivers/antennas, $r+1$, the number of tracked satellites, $s+1$, and the dimension of the baseline span, $q$. The ADOP gets smaller for larger $f, s$ or $r$, and smaller $q$. For $r=q$, the ADOP expression (60) reduces to that of an unconstrained array or small multi-frequency GNSS network. In that case the effect of the code-phase variance ratio in (60) cannot be damped by selecting a larger $r$. Hence, in the unconstrained case, the number of baselines has only a marginal effect on the network's ambiguity resolution capabilities. This situation changes significantly, however, for the case of the affine-constrained GNSS attitude model. Since the affine-constrained ADOP is symmetric in $s$ and $r$, one can now 'trade' satellites for receiver/antennas and improve the ambiguity resolution performance significantly by equipping the array with more antennas.

Once ambiguity resolution has been successful, the ILS solution $\check{Z}_{c}=\arg \min _{Z \in \mathbb{Z}^{f s \times r}} J(Z)$ can be used to determine a precise platform attitude. Different options are available to the user. The optimal one is to compute the attitude matrix as

$\check{R}=\arg \min _{R \in \mathbb{O}^{3 \times q}}\left\|\operatorname{vec}\left(\hat{R}_{c}\left(\check{Z}_{c}\right)-R\right)\right\|_{Q_{\hat{R}_{c}(Z) \hat{R}_{c}(Z)}^{2}}^{2}$

This solution has the orthonormality constraints enforced and it uses all available information, since it is based on $\hat{R}_{c}\left(\check{Z}_{c}\right)$ and its variance matrix $Q_{\hat{R}_{c}(Z) \hat{R}_{c}(Z)}$. An easier to compute, but less precise, attitude solution is obtained, if one uses the non-orthonormal estimate $\hat{R}_{c}\left(\check{Z}_{c}\right)$ directly for the computation of heading, elevation and bank (cf. 3). This approach is permitted if the precision of $\hat{R}_{c}\left(\check{Z}_{c}\right)$ is already good enough for the application at hand. This will often be the case, since the ambiguity resolved attitude solution is driven by the very precise phase measurements. The consequence of not having the orthonormality constraints enforced is, however, that inconsistency in the attitude solution may occur if other entries of $\hat{R}_{c}\left(\breve{Z}_{c}\right)$, then the ones used in (3), are used for determining the attitude parameters. A complete description of the PDF of $\hat{R}_{c}(\check{Z})$ can be obtained using the results of (Teunissen 2001). In the Gaussian case, this PDF will be a multi-modal distribution that converges to a multivariate normal distribution for success-rates approaching one.

Acknowledgments The author is a recipient of the Australian Research Council Federation Fellowship (project number FF0883188). This support is gratefully acknowledged.

Open Access This article is distributed under the terms of the Creative Commons Attribution Noncommercial License which permits any noncommercial use, distribution, and reproduction in any medium, provided the original author(s) and source are credited.

\section{Appendix}

Proof of Lemma 1 (Orthonormal matrix parametrization) $(\Rightarrow)$ Since $B S=0$ and $B T=I_{q}$, it follows from (18) that $X[S, T]=[0, R]$. With $R \in \mathbb{O}^{3 \times q}$ and thus $R^{\mathrm{T}} R=I_{q}$, the result (19) follows. $(\Leftarrow)$ If we take as right-inverse of $B$ the $r \times q$ matrix $T=B^{\mathrm{T}}\left(B B^{\mathrm{T}}\right)^{-1}$, then $T$ is a basis matrix of the range space of $B^{\mathrm{T}}$. Since $S$ is a basis matrix of the null space of $B$ and therefore of the orthogonal complement of the range space of $B^{\mathrm{T}}$, it follows that matrix $[S, T]$ is square and invertible with $S^{\mathrm{T}} T=0$. The inverse is given as $[S, T]^{-1}=\left[S\left(S^{\mathrm{T}} S\right)^{-1}, T\left(T^{\mathrm{T}} T\right)^{-1}\right]^{\mathrm{T}}$. From (19) follows that we may write $X[S, T]=[0, R]$ for some $R \in \mathbb{O}^{3 \times q}$. Hence, $X=[0, R][S, T]^{-1}=R\left(T^{\mathrm{T}} T\right)^{-1} T^{\mathrm{T}}$, which, after substitution of $T=B^{\mathrm{T}}\left(B B^{\mathrm{T}}\right)^{-1}$, gives (18) again.

Proof of Lemma 2 (Multivariate Orthogonal Decomposition) With the orthogonal projectors $P_{[M, N]}=P_{M}+P_{\bar{N}}, P_{M}=$ $M\left(M^{\mathrm{T}} Q^{-1} M\right)^{-1} M^{\mathrm{T}} Q^{-1}, P_{[M, N]}^{\perp}=I-P_{[M, N]}$ and $P_{\bar{N}}=$ $\bar{N}\left(\bar{N}^{\mathrm{T}} Q^{-1} \bar{N}\right)^{-1} \bar{N}^{\mathrm{T}} Q^{-1}$, where $\bar{N}=\left[I_{2 f s}-P_{M}\right] N$, we have

$$
\begin{aligned}
& \|\operatorname{vec}(Y-M X-N Z)\|_{Q_{Y Y}}^{2}=\left\|\operatorname{vec}\left(P_{[M, N]}^{\perp} Y\right)\right\|_{Q_{Y Y}}^{2} \\
& \quad+\left\|\operatorname{vec}\left(P_{[M, N]}[Y-M X-N Z]\right)\right\|_{Q_{Y Y}}^{2}=\|\operatorname{vec}(\hat{E})\|_{Q_{Y Y}}^{2} \\
& \quad+\left\|\operatorname{vec}\left(P_{[M, N]}[Y-M X-N Z]\right)\right\|_{Q_{Y Y}}^{2}
\end{aligned}
$$

and

$$
\begin{aligned}
& \left\|\operatorname{vec}\left(P_{[M, N]}[Y-M X-N Z]\right)\right\|_{Q_{Y Y}}^{2} \\
& =\left\|\operatorname{vec}\left(P_{\bar{N}}[Y-N Z]\right)\right\|_{Q_{Y Y}}^{2} \\
& \quad+\left\|\operatorname{vec}\left(P_{M}[Y-M X-N Z]\right)\right\|_{Q_{Y Y}}^{2}
\end{aligned}
$$




$$
\begin{aligned}
& =\|\operatorname{vec}(\bar{N}[\hat{Z}-Z])\|_{Q_{Y Y}}^{2}+\|\operatorname{vec}(M[\hat{X}(Z)-X])\|_{Q_{Y Y}}^{2} \\
& =\|\operatorname{vec}(\hat{Z}-Z)\|_{Q_{\hat{Z} \hat{Z}}}^{2}+\|\operatorname{vec}(M[\hat{X}(Z)-X])\|_{Q_{Y Y}}^{2}
\end{aligned}
$$

from which the result (38) follows.

Proof of Theorem 1 (The Constrained ADOP) Let $\hat{a}, \hat{b}$ be the LS-solution of the linear model $\mathrm{E}(y)=A a+B b, \mathrm{D}(y)=$ $Q_{y y}, a \in \mathbb{R}^{n}, b \in \mathbb{R}^{p}$. Then it follows from the determinant factorization property, see e.g. (Koch 1999), that the determinant of the variance-covariance matrix of $\hat{a}$ and $\hat{c}=C \hat{b}$ can be written as

$\left|\left[\begin{array}{cc}Q_{\hat{a} \hat{a}} & Q_{\hat{a} \hat{c}} \\ Q_{\hat{c} \hat{a}} & Q_{\hat{c} \hat{c}}\end{array}\right]\right|=\left|Q_{\hat{a} \hat{a}}\right|\left|Q_{\hat{c} \hat{c} \mid a}\right|=\left|Q_{\hat{a} \hat{a} \mid c}\right|\left|Q_{\hat{c} \hat{c}}\right|$

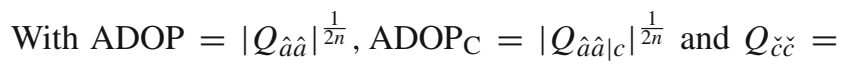
$Q_{\hat{c} \hat{c} \mid a}$, the result follows.

Proof of Theorem 2 (The Affine Constrained ADOP) Part 1 First we prove the general expression (54). Since we have Theorem 1, it suffices to derive an expression for ADOP $\mathrm{UC}_{\mathrm{UC}}$. For the determinant of the $f s r \times f s r$ ambiguity variance matrix $Q_{\hat{Z} \hat{z}}=\frac{1}{2} P \otimes Q_{\hat{z} \hat{z}}$ (cf. 29), we have

$$
\left|Q_{\hat{Z} \hat{Z}}\right|=\left(\frac{1}{2}\right)^{f s r}\left|D_{r}^{\mathrm{T}} Q_{r} D_{r}\right|^{f s}\left|Q_{\hat{z} \hat{z}}\right|^{r}
$$

where use is made of $P=D_{r}^{\mathrm{T}} Q_{r} D_{r}$ (cf. 9). From an application of the determinant factorization rule, see e.g. (Koch 1999), to the variance-covariance matrix of $\left(\hat{z}^{\mathrm{T}}, \hat{x}^{\mathrm{T}}\right)^{\mathrm{T}}$, we obtain the identity $\left|Q_{\hat{z} \hat{z}}\right|\left|Q_{\hat{x} \hat{x} \mid z}\right|=\left|Q_{\hat{z} \hat{z} \mid x}\right|\left|Q_{\hat{x} \hat{x}}\right|$ and thus

$\left|Q_{\hat{z} \hat{z}}\right|=\left|Q_{\hat{z} \hat{z} \mid x}\right| \frac{\left|Q_{\hat{x} \hat{x}}\right|}{\left|Q_{\hat{x} \hat{x} \mid z}\right|}$

Since $Q_{\hat{z}(x) \hat{z}(x)}=2\left(N^{\mathrm{T}} Q^{-1} N\right)^{-1}$, it follows with $N=$ $\left[\Lambda^{\mathrm{T}} \otimes I_{s}, 0\right]^{\mathrm{T}}$ and $Q=\operatorname{blockdiag}\left[Q_{f} \otimes D_{s}^{\mathrm{T}} Q_{\phi} D_{s}, Q_{f} \otimes\right.$ $\left.D_{s}^{\mathrm{T}} Q_{p} D_{s}\right]$, that $Q_{\hat{z}(x) \hat{z}(x)}=2\left[\left(\Lambda^{\mathrm{T}} Q_{f}^{-1} \Lambda\right) \otimes\left(D_{s}^{\mathrm{T}} Q_{\phi}\right.\right.$ $\left.\left.D_{s}\right)^{-1}\right]^{-1}$. Hence, its determinant is given as

$$
\left|Q_{\hat{z}(x) \hat{z}(x)}\right|=2^{f s}\left|\Lambda^{\mathrm{T}} Q_{f}^{-1} \Lambda\right|^{-s}\left|D_{s}^{\mathrm{T}} Q_{\phi} D_{s}\right|^{f}
$$

From (65), (66), (67) and $\left|\Lambda Q_{f}^{-1} \Lambda\right|=|\Lambda|^{2}\left|Q_{f}\right|^{-1}=$ $\bar{\lambda}^{2 f}\left|Q_{f}\right|^{-1}$, we obtain the unconstrained ADOP as

$\operatorname{ADOP}_{\mathrm{UC}}=\sqrt{\left|Q_{\hat{Z} \hat{Z}}\right|^{\frac{1}{f s r}}}=\frac{v_{\phi}}{\bar{\lambda}}\left(\sqrt{\frac{\left|Q_{\hat{x} \hat{x} \mid}\right|}{\left|Q_{\hat{x} \hat{x} \mid z}\right|}}\right)^{\frac{1}{f s}}$

with $v_{\phi}^{2}=\left|D_{r}^{\mathrm{T}} Q_{r} D_{r}\right|^{\frac{1}{r}}\left|Q_{f}\right|^{\frac{1}{f}}\left|D_{s}^{\mathrm{T}} Q_{\phi} D_{s}\right|^{\frac{1}{s}}$ and $\bar{\lambda}=$ $\left(\prod_{i=1}^{f} \lambda_{i}\right)^{\frac{1}{f}}$. Substitution of (68) into (53) gives (54).

Part 2 Now we prove the simplified expression $\left|Q_{\hat{x} \hat{x}}\right| /$ $\left|Q_{\hat{x}(z) \hat{x}(z)}\right|=\left(1+1 / \epsilon^{2}\right)^{3}$. From $Q_{\hat{x} \hat{x}}=2\left(\bar{M}^{\mathrm{T}} Q^{-1} \bar{M}\right)^{-1}$ (cf. 27) and $Q_{\hat{x}(z) \hat{x}(z)}=2\left(M^{\mathrm{T}} Q^{-1} M\right)^{-1}$ (cf. 34), it follows with $M=\left[e_{2 f} \otimes G\right], G=D_{s}^{\mathrm{T}} H, Q=\operatorname{blockdiag}\left[Q_{f} \otimes\right.$
$\left.D_{s}^{\mathrm{T}} Q_{\phi} D_{s}, Q_{f} \otimes D_{s}^{\mathrm{T}} Q_{p} D_{s}\right]$ and $\bar{M}=P_{N}^{\perp} M=\left[0, e_{f}^{\mathrm{T}} \otimes\right.$ $\left.H^{\mathrm{T}} D_{s}\right]^{\mathrm{T}}$, that

$\frac{\left|Q_{\hat{x} \hat{x}}\right|}{\left|Q_{\hat{x}(z) \hat{x}(z)}\right|}=\left|I_{3}+\left[H^{\mathrm{T}} U_{\phi} H\right]\left[H^{\mathrm{T}} U_{p} H\right]^{-1}\right|$

where $U_{\phi}=D_{s}\left(D_{s}^{\mathrm{T}} Q_{\phi} D_{s}\right)^{-1} D_{s}^{\mathrm{T}}, U_{p}=D_{s}\left(D_{s}^{\mathrm{T}} Q_{p}\right.$ $\left.D_{s}\right)^{-1} D_{s}^{\mathrm{T}}$ and $H$ is the design matrix of the undifferenced receiver-satellite geometry. Hence, if $Q_{\phi}=\epsilon^{2} Q_{p}$, then $U_{\phi}=\epsilon^{-2} U_{p}$ and the result follows.

Part 3 To prove the simplified expression for $v_{\phi}^{2}$, it is sufficient to show that

$\left|D^{\mathrm{T}} Q D\right|=\frac{\sum_{i=1}^{n} d_{i}}{\prod_{i=1}^{n} d_{i}}$

for any $(n-1) \times n$ differencing matrix $D^{\mathrm{T}}$ and any $n \times n$ diagonal matrix $Q^{-1}=\operatorname{diag}\left[d_{1}, \ldots, d_{n}\right]$. Define the $n \times n$ matrix $F=[D, e]$ in which $e$ is the $n$-vector of ones. Thus $D^{\mathrm{T}} e=$ 0 . Then according to the determinant factorization rule, see e.g. (Koch 1999), we have $\left|F^{\mathrm{T}} Q F\right|=\mid D^{\mathrm{T}} Q D \| \mathrm{e}^{\mathrm{T}} Q e-$ $\mathrm{e}^{\mathrm{T}} Q D\left(D^{\mathrm{T}} Q D\right)^{-1} D^{\mathrm{T}} Q e \mid$. This can be simplified to

$\left|F^{\mathrm{T}} Q F\right|=\left|D^{\mathrm{T}} Q D\right|\left(\mathrm{e}^{\mathrm{T}} e\right)^{2} /\left(\mathrm{e}^{\mathrm{T}} Q^{-1} e\right)$

with the use of the projector identity $Q D\left(D^{\mathrm{T}} Q D\right)^{-1} D^{\mathrm{T}}=$ $I_{n}-e\left(\mathrm{e}^{\mathrm{T}} Q^{-1} e\right)^{-1} \mathrm{e}^{\mathrm{T}} Q^{-1}$. For $Q=I_{n}$, we have from (71) that $\left|F^{\mathrm{T}} F\right|=\left|D^{\mathrm{T}} D\right|\left(\mathrm{e}^{\mathrm{T}} e\right)$. This combined with (71), shows that

$|Q|=\frac{\left|F^{\mathrm{T}} Q F\right|}{\left|F^{\mathrm{T}} F\right|}=\frac{\left|D^{\mathrm{T}} Q D\right|}{\left|D^{\mathrm{T}} D\right|} \frac{\mathrm{e}^{\mathrm{T}} e}{\mathrm{e}^{\mathrm{T}} Q^{-1} e}$

Since $D^{\mathrm{T}} D=I_{n-1}+e^{\prime} e^{\prime \mathrm{T}}$, with $e^{\prime}$ being the $(n-1)$-vector of ones, it follows that $\left|D^{\mathrm{T}} D\right|=\left|I_{n-1}+e^{\prime} e^{\prime \mathrm{T}}\right|=\left|1+e^{\prime \mathrm{T}} e^{\prime}\right|=$ $n$. Hence, $\left|D^{\mathrm{T}} D\right|=\mathrm{e}^{\mathrm{T}} e=n$. This combined with (72), shows that

$\left|D^{\mathrm{T}} Q D\right|=|Q| \mathrm{e}^{\mathrm{T}} Q^{-1} e=\frac{\mathrm{e}^{\mathrm{T}} Q^{-1} e}{\left|Q^{-1}\right|}$

This result holds for any invertible $Q$. For a diagonal matrix $Q^{-1}=\operatorname{diag}\left[d_{1}, \ldots, d_{n}\right]$, the result (70) follows.

\section{References}

Axelrad P, Ward L (1994) On-orbit gps based attitude and antenna baseline estimation. Proc ION-NTM 441-450

Bar-Itzhack IY, Montgomery P, Garrick J (1998) Algorithm for attitude determination using global positioning system. J Guid Control Dyn 21(6):846-852

Buist PJ, Teunissen PJG, Giorgi G, Verhagen S (2010) Multivariate bootstrapped relative positioning of spacecraft using GPS L1/Galileo E1 signals. Adv Space Res 47(5):770-785

Caporali A (2001) Basic direction sensing with GPS. GPS World 12(3):44-50

Chun C, Park FC (1995) Dynamics-based attitude determination using the global positioning system. J Guid Control Dyn 24(3):466-473

Cohen CE (1996) Attitude determination, global positioning system: theory and applications. In: Parkinson BW, Spilker JJ (eds) Reston, VA: AIAA, pp 519-538 
Cohen CE, Parkinson BW (1992) Integer ambiguity resolution of the GPS carrier for spacecraft attitude determination. Adv Astronaut Sci 78(8):91-118

Cohen CE, Lightsey EG, Parkinson BW (1994) Space flight tests of attitude determination using GPS. Int J Satellite Commun 12:427-433

Corbett S (1993) GPS for attitude determination and positioning in airborne remote sensing. In: Proceedings of 6th ITM ION GPS, pp 789-796

Crassidis JL, Markley FL, Lightsey EG (1999) Global positioning system integer ambiguity resolution without attitude knowledge. AIAA J Guid Control Dyn 22(2):212-218

Dai L, Ling KV, Nagarajan N (2004) Real-time attitude determination for microsatellite by LAMBDA method combined with kalman filtering. In: Proceedings 22nd AIAA international communications satellite systems conference and exhibit 2004 (ICSSC), Monterey, California, USA, p 5

DeLorenzo DS, Alban S, Gautier J, Enge P (2004) GPS attitude determination for a JPALS Testbed: Integer initialization and testing. In: Proceedings of position location and navigation symposium PLANS 2004, pp 762-770

Furuno (2003) Model SC-120: Satellite Compass. http:// wwwfurunocojp/english/indexhtml

Giorgi G, Teunissen PJG, Verhagen S, Buist PJ (2010) Testing a new multivariate GNSS carrier phase attitude determination method for remote sensing platforms. Adv Space Res 46(2):118129

Giorgi G, Teunissen PJG, Verhagen S, Buist PJ (2011) Instantaneous ambiguity resolution in GNSS-based attitude determination applications: the MC-LAMBDA method. J Guid Control Dyn, pp 1-12

Harville DA (1997) Matrix Algebra From A Statistician’s Perspective. Springer, New York

Hauschild A, Montenbruck O (2007) GPS based attitude determination for microsatellite. In: Proceedings of ION GNSS, Forth Worth, TX, USA, p 11

Hauschild A, Grillmayer G, Montenbruck O, Markgraf M, Vorsmann P (2008) GPS based attitude determination for the flying laptop satellite. Small Satellites for Earth Observation, pp 211-220. Springer, Netherlands

Hide C, Pinchin DJ (2007) Development of a low cost multiple GPS antenna attitude system. In: Proceedings of ION GNSS, pp 88-95

Hofmann-Wellenhof B, Lichtenegger H, Wasle E (2008) GNSS global navigation systems; GPS, GLONASS, Galileo and More. Springer, Berlin

Ji S, Chen W, Zhao C, Ding X, Chen Y (2007) Single epoch ambiguity resolution for galileo with the CAR and LAMBDA methods. GPS Solut 11:259-268

Jonkman N, Teunissen PJG (2001) Will geometry-free full ambiguity resolution be possible at all for long baselines? In; Proc NTM ION-GPS 2001, pp 271-280

Koch KR (1999) Parameter estimation and hypothesis testing in linear models. Springer, Berlin

Kuipers JB (2002) Quaternions and rotation sequences. Princeton University Press, Princeton

Kuylen LV, Boon F, Simsky A (2005) Attitude determination methods used in the PolarRx2@multi-antenna GPS receiver. In: Proceedings of ION GPS-2005, Long Beach, US, p 11

Kuylen LV, Nemry P, Boon F, Simsky A, Lorga JFM (2006) Comparison of attitude performance for multi-antenna receivers. Eur $\mathrm{J}$ Navig 4(2):1-9

Lachapelle G, Cannon ME, Loncarevic B (1996) Shipborne GPS attitude determination during MMST-93. IEEE J Ocean Eng 21(1):100-105

Lee HK, Wang J, Rizos C (2010) An integer ambiguity resolution procedure for GPS/pseudolite/INS integration. J Geod 79:242-255

Leick A (2004) GPS satellite surveying. Wiley, New York
Li Y, Zhang K, Roberts C, Murata M (2004) On-the-fly GPS-based attitude determination using single- and double-differenced carrier phase measurements. GPS Solut 8:93-102

Lin D, Voon LK, Nagarajan N (2004) Real-time attitude determination for microsatellite by LAMBDA method combined with kalman filtering. In: 22nd AIAA International Communication Satellite Systems Conference, Monterey, US, p 8

Lopes R (2002) Integer ambiguity resolution for spacecraft attitude determination using GPS. In: Proceedings of ION GPS, Portland, OR, US, pp 1088-1093

Lu G (1995) Development of a GPS multi-antenna system for attitude determination. UCGE Reports 20073, Dept of Geomatics Eng, University of Calgary

Madsen J, Lightsey EG (2004) Robust spacecraft attitude determination using global positioning system receivers. J Spacecr Rockets 41(4):635-643

Magnus JR, Neudecker H (1995) Matrix differential calculus with applications in statistics and econometrics. Wiley, New York

Monikes R, Wendel J, Trommer GF (2005) A modified LAMBDA method for ambiguity resolution in the presence of position domain constraints. In: Proceedings of ION GPS-2005, pp 81-87

Ong R (2010) Reliability of combined GPS/GLONASS ambiguity resolution. PhD Thesis, University of Calgary

Park C, Teunissen PJG (2003) A new carrier phase ambiguity estimation for GNSS attitude determination systems. In: Proceedings of International GPS/GNSS Symposium, Tokyo, p 8

Park C, Teunissen PJG (2009) Integer least squares with quadratic equality constraints and its application to GNSS attitude determination systems. Int J Control Autom Syst 7(4):566-576

Peng HM, Chang FR, Wang LS (1999) Attitude determination using GPS carrier phase and compass data. In: Proceedings of ION NTM 99, pp 727-732

Pinchin J (2008) Enhanced integer bootstrapping for single frequency GPS attitude determination. In: Proceedings of 21 st ITM ION GPS, pp 1290-1298

Psiaki ML (2006) Batch algorithm for global-positioning-system attitude determination and integer ambiguity resolution. J Guid Control Dyn 29(5):1071-1079

Scherzinger BM (2000) Precise robust positioning with inertial/GPS RTK. In: Proceedings of The Institute of Navigations ION GPS2000, pp 155-162

Scherzinger BM (2001) Inertially-Aided RTK position measurement. In: Proceedings of the International Symposium on Kinematic Systems in Geodesy, Geomatics and Navigation, Department of Geomatics Engineering, The University of Calgary, pp 265272

Schonemann PH (1966) A generalized solution of the orthogonal procrustes problem. Psychometrika 31(1):1-10

Simsky A, Kuylen LV, Boon F (2005) Single-board attitude determination system based on the PolaRx2@ GPS Receiver. In: Proceedings of ENC GNSS 2005, Munich, Germany, p 23

Skaloud J (1998) Reducing the GPS ambiguity search space by including inertial data. In: Proceedings of The Institute of Navigations ION GPS-98, pp 2073-2080

Strang G, Borre K (1997) Linear algebra, geodesy, and GPS. Wellesley-Cambridge Press, Wellesley

Teunissen PJG (1990) Nonlinear least-squares. Manuscripta Geodaetica $15(3): 137-150$

Teunissen PJG (1995) The least-squares ambiguity decorrelation adjustment: a method for fast GPS integer ambiguity estimation. J Geod 70:65-82

Teunissen PJG (1997) A canonical theory for short GPS baselines. Part I: The Baseline Precision; Part II: The Ambiguity Precision and Correlation; Part III: The Geometry of the Ambiguity Search Space; Part IV: Precision versus Reliability. J Geod 71(6):32-336, 389_ 401, 486-501, 513-525 
Teunissen PJG (1998) Success probability of integer GPS ambiguity rounding and bootstrapping. J Geod 72:606-612

Teunissen PJG (1999) An optimality property of the integer leastsquares estimator. J Geod 73(11):587-593

Teunissen PJG (2001) The probability distribution of the ambiguity bootstrapped GNSS baseline. J Geod 75:267-275

Teunissen PJG (2010) Integer Least Squares Theory for the GNSS Compass. J Geod (83):1-15

Teunissen PJG, Odijk D (1997) Ambiguity dilution of precision: definition, properties and application. In: Proc of ION GPS-1997, Kansas City MO, pp 891-899

Teunissen PJG, De Jonge PJ, Tiberius CCJM (1996) The volume of the GPS ambiguity search space and its relevance for integer ambiguity resolution. In: Proceedings of of ION GPS-1996, Kansas City MO, pp 889-898

Teunissen PJG, Giorgi G, Buist PJ (2011) Testing of a new single-frequency GNSS carrier phase attitude determination method: land, ship and aircraft experiments. GPS Solut 15(1):15-28

Tu CH, Tu KY, Chang FR, Wang LS (1996) GPS compass: a novel navigation equipment. IEEE Trans Aerosp Electron Syst 33(3):10631068

Unwin M, Purivigraipong S, da Silva Curiel A, Sweeting M (2002) Stand-alone spacecraft attitude determination using real flight GPS data from UOSAT-12. Acta Astronautica 51(1-9):261268

Verhagen S (2005) On the reliability of integer ambiguity resolution. Navigation 52(2):99-110
Verhagen S, Teunissen PJG (2006) New global navigation satellite system ambiguity resolution method compared to existing approaches. J Guid Control Dyn 29(4):981-991

Verhagen S, Odijk D, Teunissen PJG, Huisman L (2010) Performance improvement with low-cost multi-GNSS receivers. In: Proceedings Navitec 2010, ESA-Noordwijk, Netherlands, pp 1-8

Vollath U, Sauer K, Amarillo F, Pereira J (2003) Three or four carriers: how many are enough? In: Proc of ION GPS-2003, pp 1-8

Wahba G (1965) Problem 65-1: a least squares estimate of spacecraft attitude. SIAM Rev 7(3):384-386

Wang B, Miao L, Wang S, Shen J (2009) A constrained LAMBDA method for GPS attitude determination. GPS Solut 13:97-107

Wang C, Walker RA, Feng Y (2001) LAMBDA method for rigid body attitude determination based on GPS. Acta Aeronautica et Astronautica Sinica 22(1):61-63

Wang J, Lee HK, Lee YJ, Musa T, Rizos C (2005) Online stochastic modelling for network-based GPS real-time kinematic positioning. J Global Position Syst 4(1-2):113-119

Wertz JR (1984) Spacecraft attitude determination and control. Kluwer, Dordrecht

Yoon S, Lundberg JB (2002) An integer ambiguity resolution algorithm for real-time GPS attitude determination. Appl Math Comput 129:21-41

Ziebart M, Cross P (2003) LEO GPS attitude determination algorithm for a macro-satellite using boom-arm deployed antennas. GPS Solut 6:242-256 